MATHEMATICS OF COMPUTATION

Volume 80, Number 276, October 2011, Pages 2071-2096

S 0025-5718(2011)02469-5

Article electronically published on April 14, 2011

\title{
TREATMENT OF INCOMPATIBLE INITIAL AND BOUNDARY DATA FOR PARABOLIC EQUATIONS IN HIGHER DIMENSION
}

\author{
QINGSHAN CHEN, ZHEN QIN, AND ROGER TEMAM
}

\begin{abstract}
A new method is proposed to improve the numerical simulation of time dependent problems when the initial and boundary data are not compatible. Unlike earlier methods limited to space dimension one, this method can be used for any space dimension. When both methods are applicable (in space dimension one), the improvements in precision are comparable, but the method proposed here is not restricted by dimension.
\end{abstract}

\section{INTRODUCTION}

When performing large scale numerical simulations for evolutionary problems, we use most often initial and boundary conditions provided by approximations, by other simulations, or by experimental measurements. These data may not satisfy certain compatibility conditions verified by the solutions; thus various modifications deemed nonessential are made on the data to overcome these difficulties. Such issues are extensively addressed in the literature; see for instance in geophysical fluid mechanics 3 or 27] which contains many allusions to this difficulty; in classical fluid mechanics, see e.g. [7, 12, 13, 15, 16]; see also [1] in chemistry and [28] in a general mathematical context.

We want to address here a less known difficulty of "mathematical" nature which, the specialists believe, will become very important as we move to high resolution methods thanks to the increase of computing power and memory capacity of the computers. A very simple example of such a difficulty appears when solving in space dimension one on $(0,1)$, the heat equation $u_{t}-u_{x x}=0$ with boundary conditions $u(0, t)=u(1, t)=0$ and initial condition $u(x, 0)=1$. The solution exists and is unique (for $t>0$ ) and the analytic expressions of $u$ are provided in the literature (see e.g. 4). This problem is simple enough that it can be solved satisfactorily by numerical methods, but the solution does display singularities in the corner $x=0, t=0$ and $x=1, t=0$. For this problem and for general parabolic equations, it is known from semi-group theory [14, 22, or by using the analyticity in time of the solutions (see [11) that certain norms of $\partial u / \partial t$ grow as a power of $1 / t$ when $t \rightarrow 0$. It is believed that such singularities will affect large scale computations as our demand for better results increases. In fact, it has been observed by some authors that, when using spectral methods for the space discretization, the spectral accuracy is lost if nothing is made to address this singularity and a series of works

Received by the editor February 18, 2010 and, in revised form, July 16, 2010.

2010 Mathematics Subject Classification. Primary 35K20; Secondary 65M06.

(C)2011 American Mathematical Society 
resulted from this observation; see e.g. [2, 3, 8, 9, 10, see also [5] for a nonlinear equation.

The mathematical difficulty studied in detail, e.g., in [18, 19, 20, 23, 24, 25] is the following; even if the initial and boundary data of an evolution problem are given $\mathcal{C}^{\infty}$, the solution may not be $\mathcal{C}^{\infty}$ near $t=0$. In fact, $k$ compatibility conditions between the data are needed for the solution to be $\mathcal{C}^{k}$ near $t=0$ and hence an infinite number of compatibility conditions are needed for the solution to be $\mathcal{C}^{\infty}$. Furthermore, the initial and boundary conditions that are compatible form a relatively small set (in an informal sense), so that most numerical simulations are done with data which are not compatible, generating a loss of accuracy near $t=0$ if nothing is done. In the works mentioned above, methods have been proposed to address the first or the first two incompatibilities. It is believed that dealing with one or two incompatibilities substantially improves the quality of the simulation and, in many cases, dealing with more incompatibility conditions may become impractical. However, a strict limitation of these works is that the proposed methods only apply to space dimension one and, to the best of our knowledge, there is (there was) no method available in dimension two or larger to address this difficulty.

As we said, past works, on the computational side, have been devoted to space dimension one. This problem has been addressed in a series of articles by Flyer, Boyd, Fornberg and Swarztrauber 2, 10, 8, 9, who proposed a number of remedies in space dimension one for linear equations. Nonlinear equations in space dimension one were considered in 5 .

In these articles, the authors introduce a correction term in the linear and nonlinear cases by setting

$$
u=v+S,
$$

where $S$ absorbs the incompatibilities between the initial and boundary data up to a certain order. Now, free of incompatibilities of lower orders (the most severe ones), $v$ is computed by an appropriate numerical procedure, such as finite differences, the Galerkin finite element method, spectral or pseudo-spectral methods. As a final step, the original solution $u$ is recovered through (1.1). This remedy procedure effectively reduces the errors at the spatio-temporal corners during the short initial transient period.

For dimensions higher than one, the construction of $S$, to correct for singularities generated at $t=0$ by incompatible data, remains an open problem. A method to overcome this difficulty is proposed, analyzed and tested in this article.

In this article, we intend to study, from both a theoretical and numerical point of view, the incompatibility issue for the multi-dimensional time-dependent linear parabolic equation:

$$
\left\{\begin{array}{l}
u_{t}-\nu \triangle u=f, \quad x \in \Omega \subset R^{d}, \quad t \in R^{+}, \\
\left.u\right|_{t=0}=u_{0}, \\
\left.u\right|_{\partial \Omega}=g .
\end{array}\right.
$$

We believe that our method applies to a more general parabolic equation, but we restrict ourselves to equation (1.2) in this article devoted to feasibility.

The method that we propose is based on the concept of penalty. We replace in (1.2) the boundary value $\left.u\right|_{\partial \Omega}=g$ by $\left.u\right|_{\partial \Omega}=k^{\varepsilon}$. This boundary value $k^{\varepsilon}$ which depends on a parameter $\varepsilon>0$ is such that $\left.k^{\varepsilon}\right|_{t=0}=\left.u_{0}\right|_{\partial \Omega}$ (see equations (2.1), 
(2.2) below), so that the first incompatibility has disappeared. Now $k^{\varepsilon}$, through a penalty procedure with parameter $\varepsilon$, is forced to rapidly vary from $\left.u_{0}\right|_{\partial \Omega}$ at $t=0$ to the desired value, namely $g$. This is achieved through equation (2.2); initially $k_{t}^{\varepsilon}$ is large, but it becomes rapidly of order 1 , and then, by the first equation (2.2), $k^{\varepsilon}-g$ is of order $\varepsilon$. It is easy of course to integrate equation (2.2) although an explicit solution is not available in the general case where $g$ depends on time. The concept of penalty has been introduced in the mathematical literature by R. Courant 6 ; it has been adapted to evolution problems by J. L. Lions in 21, a reference which contains many evolution equations similar to (2.2) (Chapter 3, Sections 5 to 8); it is widely used in optimization 1 ; see also 26] (Chapter 1, Section 6). In this work, we first present our approach in detail and study it theoretically to prove the strong convergence of the method. Then we implement it numerically on a number of examples. Because the penalty method does not depend on the properties of $\Omega$, we believe that this method can be applied to many systems with many different domains $\Omega$. The question that remains is the choice of small $\varepsilon$. In optimization theory, the choice of $\varepsilon$ is usually made by trial and error and is not a major issue. It does not follow the "intuitive" idea that the error becomes smaller as $\varepsilon$ becomes smaller because of many other contingent errors such as round-off and descretization errors. In general the error becomes "optimal" for some value of $\varepsilon$ and the method gives fewer good results for smaller or larger values of $\varepsilon$. In our case (see Figures 6 and (7), at the initial steps, the error decreases sharply as $\varepsilon$ increases and remains close to 0 , then it becomes stable flat. At the final steps, the error increases almost linearly as $\varepsilon$ increases. With $\varepsilon$ at about 0.1 , the initial error is minimized while the error at the final step is well controlled. In a short time period $\varepsilon=0.5$ gives us smaller errors and again after a short time period $\varepsilon=0.1$ gives us a smaller errors. In general the choice of $\varepsilon$ really depends on our goals of the computation.

This article is organized as follows. In Section 2 we present the method and establish various approximation results. Then, in Section 3 we present numerical results showing the efficiency of the method and comparing it to earlier methods. In Section 4 we present some conclusions and perspective of future developments.

\section{PENALTy METHOD}

2.1. Perturbed problem (and the statement of the main result). We consider the system (1.2), where $\nu>0$. If $\left.u_{0}\right|_{\partial \Omega} \neq g(0)$, then we face an incompatibility problem, in which case we consider a new system instead, namely, for $\varepsilon>0$ fixed,

$$
\begin{gathered}
\left\{\begin{array}{l}
u_{t}^{\varepsilon}-\nu \triangle u^{\varepsilon}=f, \quad x \in \Omega \subset R^{d}, \quad t \in R^{+}, \\
\left.u^{\varepsilon}\right|_{t=0}=u_{0}, \\
\left.u^{\varepsilon}\right|_{\partial \Omega}=k^{\varepsilon}
\end{array}\right. \\
\left\{\begin{array}{l}
k_{t}^{\varepsilon}+\frac{1}{\varepsilon}\left(k^{\varepsilon}-g\right)=0, \quad t \in R^{+}, \\
k^{\varepsilon}(0)=\left.u_{0}\right|_{\partial \Omega} .
\end{array}\right.
\end{gathered}
$$

In this article, $|\cdot|$ is the $L^{2}(\Omega)$ norm, and $\|\cdot\|=|\nabla \cdot|$ is the $H_{0}^{1}(\Omega)$ norm; for other norms, we will use the subscript notation.

The system (2.1) -(2.2) is actually decoupled and (2.2) is just an Ordinary Differential Equation with $x \in \partial \Omega$ as a parameter. As we see below, if we are given $g, g^{\prime}=\frac{\partial g}{\partial t} \in L^{2}\left(0, T ; H^{\frac{1}{2}}(\Gamma)\right)$, then we have the existence and uniqueness of $k^{\varepsilon}$ in

\footnotetext{
${ }^{1}$ A search on Google with the words "optimization, penalty" produced 3,350,000 entries.
} 
$L^{2}\left(0, T ; H^{\frac{1}{2}}(\Gamma)\right)$ and furthermore, by the effect of the penalty term, $\left(k^{\varepsilon}-g\right) / \varepsilon, k^{\varepsilon}$ converges to $g$ in suitable spaces as $\varepsilon \rightarrow 0$. Equations (2.1) is a heat equation with nonhomogeneous boundary conditions, and we have the existence and uniqueness of a solution if the data are sufficiently regular. Then we have the following theorem.

Theorem 2.1. Assume that we are given $g \in L^{\infty}\left(0, T ; H^{\frac{1}{2}}(\Gamma)\right)(\Gamma=\partial \Omega)$, with $g_{t} \in L^{2}\left(0, T ; H^{\frac{1}{2}}(\Gamma)\right)$, and $u_{0} \in H^{1}(\Omega)$. Then (1.2) has a unique solution $u \in$ $L^{2}\left(0, T ; H^{1}(\Omega)\right) \cap \mathcal{C}\left([0, T] ; L^{2}(\Omega)\right)$, and for each $\varepsilon>0$, (2.1) -(2.2) has a unique solution $u^{\varepsilon} \in L^{2}\left(0, T ; H^{1}(\Omega)\right) \cap \mathcal{C}\left([0, T] ; L^{2}(\Omega)\right), k^{\varepsilon} \in L^{2}\left(0, T ; H^{\frac{1}{2}}(\Gamma)\right)$. Furthermore, as $\varepsilon \rightarrow 0$,

$$
\begin{aligned}
u^{\varepsilon} \rightarrow u \text { in } & L^{2}\left(0, T ; H^{-1}(\Omega)\right) \text { strongly, and in } \\
& \mathcal{C}\left(\left[t_{0}, T\right] ; H^{-2}(\Omega)\right) \text { strongly, } \forall t_{0}>0 .
\end{aligned}
$$

Remark 2.1. We do not prove a strong convergence of $u^{\varepsilon}$ to $u$ on all of $[0, T]$ in the $L^{\infty}$ sense, and we do not expect such a convergence to occur since $u$ has a singularity at $t=0$. Alternatively, one could capture the singularity of $u$ near $t=0$ by using the methods of singular perturbation theory, e.g., as in Jung-Temam [17, which we do briefly in Section 2.3, and will also be studied elsewhere.

Before we prove Theorem 2.1, we will first prove the following lemma.

Lemma 2.1. If $g \in L^{\infty}\left(0, T ; H^{\frac{1}{2}}(\Gamma)\right)$ and $g^{\prime} \in L^{2}\left(0, T ; H^{\frac{1}{2}}(\Gamma)\right)$, then there exists a unique $k^{\varepsilon}$ in $L^{2}\left(0, T ; H^{\frac{1}{2}}(\Gamma)\right)$ satisfying (2.2), and as $\varepsilon \rightarrow 0, k^{\varepsilon} \rightarrow g$ in $L^{2}\left(0, T ; H^{\frac{1}{2}}(\Gamma)\right)$ strongly. Furthermore, as $\varepsilon \rightarrow 0, \int_{0}^{t} k^{\varepsilon}(s) d s \rightarrow \int_{0}^{t} g(s) d s$ in $L^{2}\left(0, T ; H^{\frac{1}{2}}(\Gamma)\right)$ strongly.

Proof. We explicitly solve the ODE system (2.2), and we obtain the solution $k^{\varepsilon} \in$ $L^{2}\left(0, T ; H^{\frac{1}{2}}(\Gamma)\right)$ :

$$
k^{\varepsilon}(t)=e^{-\frac{t}{\varepsilon}} k^{\varepsilon}(0)+\int_{0}^{t} \frac{1}{\varepsilon} g(s) e^{\frac{s-t}{\varepsilon}} d s .
$$

Then we rewrite $(2.2)_{1}$ in the form

$$
\left(k^{\varepsilon}-g\right)_{t}+\frac{1}{\varepsilon}\left(k^{\varepsilon}-g\right)=-g_{t} .
$$

Taking the scalar product of (2.5) with $k^{\varepsilon}-g$ in $H^{\frac{1}{2}}(\Gamma)$, we obtain

$$
\begin{aligned}
\frac{1}{2} \frac{d}{d t}\left|k^{\varepsilon}-g\right|_{H^{\frac{1}{2}}(\Gamma)}^{2}+ & \frac{1}{\varepsilon}\left|k^{\varepsilon}-g\right|_{H^{\frac{1}{2}}(\Gamma)}^{2}=-\left(g_{t}, k^{\varepsilon}-g\right) \\
& \leq\left|g_{t}\right|_{H^{\frac{1}{2}}(\Gamma)}\left|k^{\varepsilon}-g\right|_{H^{\frac{1}{2}}(\Gamma)} \\
& \leq \frac{\varepsilon}{2}\left|g_{t}\right|_{H^{\frac{1}{2}}(\Gamma)}^{2}+\frac{1}{2 \varepsilon}\left|k^{\varepsilon}-g\right|_{H^{\frac{1}{2}}(\Gamma)}^{2} .
\end{aligned}
$$

Hence

$$
\frac{d}{d t}\left|k^{\varepsilon}-g\right|_{H^{\frac{1}{2}(\Gamma)}}^{2}+\frac{1}{\varepsilon}\left|k^{\varepsilon}-g\right|_{H^{\frac{1}{2}}(\Gamma)}^{2} \leq \varepsilon\left|g_{t}\right|_{H^{\frac{1}{2}(\Gamma)}}^{2} .
$$

Using the Gronwall inequality we obtain

$$
\left|k^{\varepsilon}-g\right|_{H^{\frac{1}{2}(\Gamma)}}^{2}(t) \leq e^{-\frac{t}{\varepsilon}}\left|k^{\varepsilon}-g\right|_{H^{\frac{1}{2}}(\Gamma)}^{2}(0)+\varepsilon\left|g_{t}\right|_{L^{2}\left(0, T ; H^{\frac{1}{2}}(\Gamma)\right)}^{2} .
$$


We integrate (2.7) over $(0, T)$, and we obtain $\left(u_{0} \in H^{1}(\Omega)\right)$.2

$$
\int_{0}^{T}\left|k^{\varepsilon}-g\right|_{H^{\frac{1}{2}}(\Gamma)}^{2} d t \leq \varepsilon\left(1-e^{-\frac{T}{\varepsilon}}\right)\left|u_{0}\right|_{\Gamma}-\left.g(0)\right|_{H^{\frac{1}{2}}(\Gamma)} ^{2}+\varepsilon T\left|g_{t}\right|_{L^{2}\left(0, T ; H^{\frac{1}{2}}(\Gamma)\right)}^{2}
$$

and hence

$$
\left|k^{\varepsilon}-g\right|_{L^{2}\left(0, T ; H^{\frac{1}{2}}(\Gamma)\right)}=O(\sqrt{\varepsilon}),
$$

which implies

$$
k^{\varepsilon} \rightarrow g \text { strongly in } L^{2}\left(0, T ; H^{\frac{1}{2}}(\Gamma)\right) \text { as } \varepsilon \rightarrow 0 .
$$

Integrating (2.5) from 0 to $t$, we obtain

$$
\int_{0}^{t}\left(k^{\varepsilon}-g\right) d s=-\varepsilon\left(k^{\varepsilon}-g\right)(t)-\varepsilon g(t)+\left.\varepsilon u_{0}\right|_{\partial \Omega},
$$

which yields

$$
\int_{0}^{t} k^{\varepsilon}(s) d s \rightarrow \int_{0}^{t} g(s) d s \text { strongly in } L^{2}\left(0, T ; H^{\frac{1}{2}}(\Gamma)\right) \text { as } \varepsilon \rightarrow 0 .
$$

The proof of Lemma 2.1 is complete.

Remark 2.2. We could prove a stronger result namely, $k^{\varepsilon} \rightarrow g, \int_{0}^{t} k^{\varepsilon}(s) d s \rightarrow$ $\int_{0}^{t} g(s) d s$ strongly in $L^{q}\left(0, T ; H^{\frac{1}{2}}(\Gamma)\right)$, for all $1 \leq q<\infty$. But in this article, $q=2$ is enough for our needs; and for $q=\infty$, from (2.7), we obtain

$$
k^{\varepsilon}-g=O(\sqrt{\varepsilon}) \text { in } L^{\infty}\left(t_{0}, T ; H^{\frac{1}{2}}(\Gamma)\right) \text { for } \forall t_{0}>0,
$$

and also from (2.10), because $k^{\varepsilon}-g$ and $g$ are bounded in $L^{\infty}\left(0, T ; H^{\frac{1}{2}}(\Gamma)\right)$, we obtain

$$
\int_{0}^{t} k^{\varepsilon}(s) d s \rightarrow \int_{0}^{t} g(s) d s \text { strongly in } L^{\infty}\left(0, T ; H^{\frac{1}{2}}(\Gamma)\right),
$$

the norm of the difference being of order $\varepsilon$.

2.2. Convergence results for $u^{\varepsilon}$. Since $\Omega$ is smooth, there exists a lifting operator $L$, linear continuous from $H^{\frac{1}{2}}(\Gamma)$ to $H^{1}(\Omega)$. We consider such an operator and set $K^{\varepsilon}=L\left(k^{\varepsilon}\right), G=L(g)$, and thus have by assumption $G \in L^{\infty}\left(0, T ; H^{1}(\Omega)\right)$, $G_{t} \in L^{2}\left(0, T ; H^{1}(\Omega)\right)$. So we immediately infer from (2.9), (2.11), (2.12) and (2.13) that, as $\varepsilon \rightarrow 0$

$$
\begin{aligned}
& K^{\varepsilon} \rightarrow G \text { strongly in } L^{2}\left(0, T ; H^{1}(\Omega)\right) \cap L^{\infty}\left(t_{0}, T ; H^{1}(\Omega)\right) \forall t_{0}>0, \\
& \int_{0}^{t} K^{\varepsilon}(s) d s \rightarrow \int_{0}^{t} G(s) d s \text { strongly in } L^{\infty}\left(0, T ; H^{1}(\Omega)\right) .
\end{aligned}
$$

We now prove Theorem 2.1 .

Proof of Theorem 2.1. Set $v^{\varepsilon}=u^{\varepsilon}-K^{\varepsilon}$; then the system (2.1) yields

$$
\left\{\begin{array}{l}
v_{t}^{\varepsilon}-\nu \Delta v^{\varepsilon}=f-K_{t}^{\varepsilon}+\nu \triangle K^{\varepsilon}, \\
\left.v^{\varepsilon}\right|_{t=0}=u_{0}-K^{\varepsilon}(0)=u_{0}-\left.L u_{0}\right|_{\partial \Omega}, \\
\left.v^{\varepsilon}\right|_{\partial \Omega}=0 .
\end{array}\right.
$$

\footnotetext{
${ }^{2}$ We do not address the question of minimal regularity of $u_{0}$, that is e.g. $u_{0} \in L^{2}(\Omega)$, which is not in the scope of this article. Indeed the problem of incompatible data occurs already with very smooth data.
} 
Integrating $(2.16)_{1}$ from 0 to $t$, we obtain

$$
v^{\varepsilon}(t)-\nu \triangle \int_{0}^{t} v^{\varepsilon}(s) d s=\int_{0}^{t} f(s) d s-K^{\varepsilon}(t)+\nu \triangle \int_{0}^{t} K^{\varepsilon}(s) d s+u_{0} .
$$

We set $V^{\varepsilon}=\int_{0}^{t} v^{\varepsilon}(s) d s$ (with $V^{\varepsilon}(0)=0$ ), and $F(t)=\int_{0}^{t} f(s) d s+u_{0}$, so $V^{\varepsilon}$ solves the following system:

$$
\left\{\begin{array}{l}
V_{t}^{\varepsilon}-\nu \triangle V^{\varepsilon}=F-K^{\varepsilon}+\nu \triangle \int_{0}^{t} K^{\varepsilon}(s) d s, \\
\left.V^{\varepsilon}\right|_{t=0}=0, \\
\left.V^{\varepsilon}\right|_{\partial \Omega}=0 .
\end{array}\right.
$$

We take the scalar product of (2.18) $)_{1}$ with $V^{\varepsilon}$ in $L^{2}(\Omega)$ and find,

$$
\frac{1}{2} \frac{d}{d t}\left|V^{\varepsilon}\right|^{2}+\nu\left\|V^{\varepsilon}\right\|^{2}=\left(F, V^{\varepsilon}\right)-\left(K^{\varepsilon}, V^{\varepsilon}\right)+\nu\left(\triangle \int_{0}^{t} K^{\varepsilon}(s) d s, V^{\varepsilon}\right) .
$$

We can bound the terms in the right-hand-side of (2.19) as follows:

$$
\begin{aligned}
&\left(F, V^{\varepsilon}\right) \leq|F|\left|V^{\varepsilon}\right| \leq c_{1}|F|\left\|V^{\varepsilon}\right\| \leq c_{1}^{\prime}|F|^{2}+\frac{\nu}{6}\left\|V^{\varepsilon}\right\|^{2} \\
&-\left(K^{\varepsilon}, V^{\varepsilon}\right) \leq\left|K^{\varepsilon}\right|\left|V^{\varepsilon}\right| \leq c_{1}\left|K^{\varepsilon}\right|\left\|V^{\varepsilon}\right\| \leq c_{2}^{\prime}\left|K^{\varepsilon}\right|^{2}+\frac{\nu}{6}\left\|V^{\varepsilon}\right\|^{2}, \\
& \nu\left(\triangle \int_{0}^{t} K^{\varepsilon}(s) d s, V^{\varepsilon}\right)=-\nu\left(\nabla \int_{0}^{t} K^{\varepsilon}(s) d s, \nabla V^{\varepsilon}\right) \\
& \leq \nu\left\|\int_{0}^{t} K^{\varepsilon}(s) d s\right\|\left\|V^{\varepsilon}\right\| \\
& \leq \frac{\nu}{6}\left\|V^{\varepsilon}\right\|^{2}+c_{3}^{\prime}\left\|\int_{0}^{t} K^{\varepsilon}(s) d s\right\|^{2}
\end{aligned}
$$

Here and below, the $c, c^{\prime}, c_{i}, c_{i}^{\prime}$ are various constants independent of $\varepsilon$, which may be different at different places.

Combining (2.19), (2.20), (2.21) and (2.22) gives

$$
\frac{d}{d t}\left|V^{\varepsilon}\right|^{2}+\nu\left\|V^{\varepsilon}\right\|^{2} \leq c_{1}^{\prime}|F|^{2}+c_{2}^{\prime}\left|K^{\varepsilon}\right|^{2}+c_{3}^{\prime}\left\|\int_{0}^{t} K^{\varepsilon}(s) d s\right\|^{2} .
$$

Integrating (2.23) over $(0, t)$, we obtain

$$
\begin{aligned}
\left|V^{\varepsilon}(t)\right|^{2}+\nu \int_{0}^{t}\left\|V^{\varepsilon}\right\|^{2} d s & \leq c_{1}^{\prime} \int_{0}^{t}|F|^{2} d s+c_{2}^{\prime} \int_{0}^{t}\left|K^{\varepsilon}\right|^{2} d s \\
& +c_{3}^{\prime} \int_{0}^{t}\left\|\int_{0}^{s} K^{\varepsilon}(\tau) d \tau\right\|^{2} d s .
\end{aligned}
$$

We also integrate (2.23) over $(0, T)$, and obtain

$$
\begin{aligned}
\left|V^{\varepsilon}(T)\right|^{2}+\nu \int_{0}^{T}\left\|V^{\varepsilon}\right\|^{2} d s & \leq c_{1}^{\prime} \int_{0}^{T}|F|^{2} d s+c_{2}^{\prime} \int_{0}^{T}\left|K^{\varepsilon}\right|^{2} d s \\
& +c_{3}^{\prime} \int_{0}^{T}\left\|\int_{0}^{s} K^{\varepsilon}(\tau) d \tau\right\|^{2} d s .
\end{aligned}
$$


It follows from (2.14) and 2.15) that $K^{\varepsilon}$ and $\int_{0}^{t} K^{\varepsilon}(s) d s$ are bounded in $L^{2}\left(0, T ; H^{1}(\Omega)\right)$, and thus (2.24), (2.25) yields:

$$
V^{\varepsilon} \text { remains bounded in } L^{\infty}\left(0, T ; L^{2}(\Omega)\right) \cap L^{2}\left(0, T ; H_{0}^{1}(\Omega)\right) \text { as } \varepsilon \rightarrow 0 .
$$

Thus, there exists a subsequence $V^{\varepsilon^{\prime}}$ and $V \in L^{\infty}\left(0, T ; L^{2}(\Omega)\right) \cap L^{2}\left(0, T ; H_{0}^{1}(\Omega)\right)$ such that, as $\varepsilon^{\prime} \rightarrow 0$,

$$
V^{\varepsilon^{\prime}} \rightarrow V \text { weakly in } L^{2}\left(0, T ; H_{0}^{1}(\Omega)\right) \text {, and weak-star in } L^{\infty}\left(0, T ; L^{2}(\Omega)\right) .
$$

Using (2.14), (2.15) and (2.27), we can pass to the limit in (2.16) with the sequence $\varepsilon^{\prime} \rightarrow 0$. We proceed as follows.

For all $a \in H_{0}^{1}(\Omega)$, and $\phi$ in $C^{1}(0, T)$ with $\phi(T)=0$, we multiply (2.18) 1 by $a \phi$ and integrate over $\Omega \times(0, T)$; we obtain

$$
\begin{array}{r}
-\int_{0}^{T}\left(V^{\varepsilon^{\prime}}, a\right) \phi^{\prime}(t) d t+\nu \int_{0}^{T}\left(\nabla V^{\varepsilon^{\prime}}, \nabla a\right) \phi(t) d t=\int_{0}^{T}(F, a) \phi(t) d t \\
-\int_{0}^{T}\left(K^{\varepsilon^{\prime}}, a\right) \phi(t) d t-\nu \int_{0}^{T}\left(\nabla \int_{0}^{t} K^{\varepsilon^{\prime}}(s) d s, \nabla a\right) \phi(t) d t .
\end{array}
$$

Passing to the limit with (2.14), (2.15), (2.27), we find

$$
\begin{aligned}
-\int_{0}^{T}(V, a) \phi^{\prime}(t) d t & +\nu \int_{0}^{T}(\nabla V, \nabla a) \phi(t) d t=\int_{0}^{T}(F, a) \phi(t) d t \\
& -\int_{0}^{T}(G, a) \phi(t) d t-\nu \int_{0}^{T}\left(\nabla \int_{0}^{t} G(s) d s, \nabla a\right) \phi(t) d t .
\end{aligned}
$$

Taking $\phi \in \mathcal{D}(0, T)$, we see that $V$ satisfies

$$
\left(V_{t}, a\right)-\nu(\triangle V, a)=\left(F-G+\nu \triangle \int_{0}^{t} G(s) d s, a\right), \quad \forall a \in H_{0}^{1}(\Omega) .
$$

Now we want to show that $V(0)=0$.

We classically integrate (2.30) times $\phi(t)$ over $(0, T)$ and we obtain:

$$
\begin{aligned}
-\int_{0}^{T}(V, a) \phi^{\prime}(t) d t & +\nu \int_{0}^{T}(\nabla V, \nabla a) \phi(t) d t=\int_{0}^{T}\left(\nu \triangle \int_{0}^{t} G(s) d s, a\right) \phi(t) d t \\
& +\int_{0}^{T}(F-G, a) \phi(t) d t+(V(0), a) \phi(0) .
\end{aligned}
$$

By comparing with (2.29), we find that

$$
(V(0), a) \phi(0)=0,
$$

for every $a \in H_{0}^{1}(\Omega)$ and every $\phi \in \mathcal{C}^{1}([0, T])$ with $\phi(T)=0$. This implies $V(0)=0$ as desired. Finally, $V$ satisfies

$$
\left\{\begin{array}{l}
V_{t}-\nu \triangle V=F-G+\nu \triangle \int_{0}^{t} G(s) d s \\
\left.V\right|_{t=0}=0 \\
\left.V\right|_{\partial \Omega}=0 .
\end{array}\right.
$$


Remark 2.3. Furthermore, we could prove that the whole sequence $V^{\varepsilon} \rightarrow V$ weakly in $L^{2}\left(0, T ; H_{0}^{1}(\Omega)\right)$, and weak star in $L^{\infty}\left(0, T ; L^{2}(\Omega)\right)$. Indeed, if not, arguing by contradiction, we could find a subsequence $\varepsilon_{i} \rightarrow 0$, such that

$$
\begin{array}{r}
V^{\varepsilon_{i}} \nrightarrow V \text { in } L^{2}\left(0, T ; H_{0}^{1}(\Omega)\right) \text { weakly, } \\
L^{\infty}\left(0, T ; L^{2}(\Omega)\right) \text { weak-star. }
\end{array}
$$

Repeating the argument above leading to (2.27), we could extract from $\varepsilon_{i}$ a subsequence $\varepsilon_{i}^{\prime}$ and find $\bar{V}$ such that, as $\varepsilon_{i}^{\prime} \rightarrow 0$,

$$
\begin{aligned}
V^{\varepsilon_{i}^{\prime}} \rightarrow \bar{V} \text { in } L^{2}\left(0, T ; H_{0}^{1}(\Omega)\right) \text { weakly, } \\
L^{\infty}\left(0, T ; L^{2}(\Omega)\right) \text { weak-star, }
\end{aligned}
$$

where $\bar{V}$ is the solution of (2.33). But the solution of (2.33) is unique; hence $V=\bar{V}$, and then (2.35) contradicts (2.34).

Before we finish the proof of the theorem, we now prove the following lemma.

Lemma 2.2. Under the assumptions of Theorem 2.1, with $V, V^{\varepsilon}$ being the solutions of (2.33) and (2.18), we have, as $\varepsilon \rightarrow 0$,

$$
V^{\varepsilon} \rightarrow \text { Vstrongly in } L^{2}\left(0, T ; H_{0}^{1}(\Omega)\right) \cap \mathcal{C}\left([0, T] ; L^{2}(\Omega)\right)
$$

Proof. We subtract $(2.18)_{1}$ from $(2.33)_{1}$, and obtain

$$
V_{t}-V_{t}^{\varepsilon}-\nu\left(\triangle V-\triangle V^{\varepsilon}\right)=K^{\varepsilon}-G+\nu\left(\triangle \int_{0}^{t} G(s) d s-\triangle \int_{0}^{t} K^{\varepsilon}(s) d s\right) .
$$

We then take the scalar product of (2.37) with $V-V^{\varepsilon}$ in $L^{2}(\Omega)$, and integrate in time from 0 to $t$, and we obtain:

$$
\begin{array}{r}
\frac{1}{2}\left|\left(V-V^{\varepsilon}\right)(t)\right|^{2}+\nu \int_{0}^{t}\left\|V-V^{\varepsilon}\right\|^{2} d s=\int_{0}^{t}\left(K^{\varepsilon}-G, V-V^{\varepsilon}\right) d s \\
\int_{0}^{t}\left(\nu \triangle \int_{0}^{t} G(s) d s-\nu \triangle \int_{0}^{t} K^{\varepsilon}(s) d s, V-V^{\varepsilon}\right) d s .
\end{array}
$$

Now we set

$$
\chi_{\varepsilon}(t)=\frac{1}{2}\left|\left(V-V^{\varepsilon}\right)(t)\right|^{2}+\frac{\nu}{2} \int_{0}^{t}\left\|V-V^{\varepsilon}\right\|^{2} d s,
$$


and estimate the right-hand side of (2.38) as follows:

$$
\begin{aligned}
& \int_{0}^{t}\left(K^{\varepsilon}-G, V-V^{\varepsilon}\right) d s \leq \int_{0}^{t}\left|K^{\varepsilon}-G\right|\left|V-V^{\varepsilon}\right| d s \\
& \leq c_{1} \int_{0}^{t}\left|K^{\varepsilon}-G\right|\left\|V-V^{\varepsilon}\right\| d s \\
& \leq c_{1}\left(\int_{0}^{T}\left|K^{\varepsilon}-G\right|^{2} d s\right)^{\frac{1}{2}}\left(\int_{0}^{t}\left\|V-V^{\varepsilon}\right\|^{2} d s\right)^{\frac{1}{2}} \\
& \leq c \int_{0}^{T}\left|K^{\varepsilon}-G\right|^{2} d s+\frac{\nu}{4} \int_{0}^{t}\left\|V-V^{\varepsilon}\right\|^{2} d s \\
& \int_{0}^{t} \nu\left(\triangle \int_{0}^{s} G(\tau) d \tau-\right.\left.\triangle \int_{0}^{s} K^{\varepsilon}(\tau) d \tau, V-V^{\varepsilon}\right) d s \\
& \leq \nu \int_{0}^{t}\left\|\int_{0}^{s}\left(G(\tau)-K^{\varepsilon}(\tau)\right) d \tau\right\|\left\|V-V^{\varepsilon}\right\| d s \\
& \leq c^{\prime} \int_{0}^{T}\left\|\int_{0}^{s}\left(G-K^{\varepsilon}\right)(\tau) d \tau\right\|^{2} d s+\frac{\nu}{4} \int_{0}^{t}\left\|V-V^{\varepsilon}\right\|^{2} d s
\end{aligned}
$$

Combining (2.40) and (2.41), we see that

$$
\chi_{\varepsilon}(t) \leq c^{\prime} \int_{0}^{T}\left\|\int_{0}^{s}\left(G-K^{\varepsilon}\right)(\tau) d \tau\right\|^{2} d s+c \int_{0}^{T}\left|K^{\varepsilon}-G\right|^{2} d s .
$$

The right-hand side of (2.42) converges to 0 as $\epsilon$ converges to 0 , because of (2.14) and (2.15), and so does $\chi_{\varepsilon}(t)$. For $t=T$, we find

$$
V^{\varepsilon} \rightarrow V \text { strongly in } L^{2}\left(0, T ; H_{0}^{1}(\Omega)\right) \text { as } \varepsilon \rightarrow 0,
$$

and taking the supreme of (2.42) with respect to $t$, we see that

$$
V^{\varepsilon} \rightarrow V \text { strongly in } L^{\infty}\left(0, T ; L^{2}(\Omega)\right) \text { as } \varepsilon \rightarrow 0 .
$$

The lemma is proved.

Now we apply Lemma 2.2 and obtain as $\varepsilon \rightarrow 0$,

$$
\triangle V^{\varepsilon} \rightarrow \triangle V \text { strongly in } L^{2}\left(0, T ; H^{-1}(\Omega)\right) \cap \mathcal{C}\left([0, T] ; H^{-2}(\Omega)\right),
$$

and from (2.15), we obtain as $\varepsilon \rightarrow 0$,

$$
\triangle \int_{0}^{t} K^{\varepsilon}(s) d s \rightarrow \triangle \int_{0}^{t} G(s) d s \text { strongly in } L^{\infty}\left(0, T ; H^{-1}(\Omega)\right),
$$

so after comparing $(2.18)_{1}$ with $(2.33)_{1}$, we conclude that as $\varepsilon \rightarrow 0$,

$$
\begin{aligned}
V_{t}^{\varepsilon} \rightarrow V_{t} \text { strongly in } L^{2}\left(0, T ; H^{-1}(\Omega)\right) \text { and } \\
\qquad \mathcal{C}\left(\left[t_{0}, T\right] ; H^{-2}\right) \quad \forall t_{0}>0 .
\end{aligned}
$$

Now we define $v=V_{t}$, and take the derivative on (2.33) ${ }_{1}$, we obtain that $v$ solves the following system:

$$
\left\{\begin{array}{l}
v_{t}-\nu \triangle v=f-G_{t}+\nu \triangle G, \\
\left.v\right|_{t=0}=u_{0}-\left.u_{0}\right|_{\partial \Omega}, \\
\left.v\right|_{\partial \Omega}=0 .
\end{array}\right.
$$


So (2.47) yields as $\varepsilon \rightarrow 0$,

$$
\begin{aligned}
& v^{\epsilon} \rightarrow v \text { strongly in } L^{2}\left(0, T ; H^{-1}(\Omega)\right) \text { and } \\
& \mathcal{C}\left(\left[t_{0}, T\right] ; H^{-2}\right) \quad \forall t_{0}>0 .
\end{aligned}
$$

The final stage of the proof of Theorem 2.1 consists of reinterpreting the results above, that is, (2.14), (2.15) and (2.49) in terms of the convergence of $u^{\varepsilon}=v^{\varepsilon}+K^{\varepsilon}$ towards $u=v+G$; we obtain precisely (2.3). Theorem 2.1 is proven.

Remark 2.4. Similarly, as Remark 2.2, we see that we also have $u^{\varepsilon} \rightarrow u$ strongly in $L^{q}\left(0, T ; H^{-1}(\Omega)\right)$ for all $1 \leq q<\infty$.

2.3. Boundary layer analysis for $k^{\varepsilon}$. In the previous section, Lemma 2.1 stated that under our assumptions, $k^{\varepsilon}$ strongly converges to $g$ in $L^{2}\left(0, T ; H^{\frac{1}{2}}(\Gamma)\right)$, as $\varepsilon \rightarrow 0$. Here in order to better compare $k^{\varepsilon}$ and $g$, we are going to study the boundary layer for the system (2.2).

Along the asymptotic analysis, we define the outer expansion $k^{\varepsilon} \sim \sum_{j=0}^{\infty} \varepsilon^{j} k^{j}$. By formal identification at each power of $\varepsilon$, we obtain

$$
\begin{aligned}
& O\left(\varepsilon^{-1}\right): \quad k^{0}=g, \\
& O\left(\varepsilon^{j}\right): \quad k_{t}^{j}+k^{j+1}=0, \quad \forall j \geq 0 .
\end{aligned}
$$

By explicit calculations, we find:

$$
k^{j}=(-1)^{j} g^{(j)}, \forall j \geq 0 .
$$

It is clear that the functions $k^{j}$ of the outer expansion do not generally satisfy the initial condition in (2.2) in the case of interest here where $g(0) \neq\left. u_{0}\right|_{\partial \Omega}$. To account for this discrepancy, we classically introduce the inner expansion $k^{\varepsilon} \sim \sum_{j=0}^{\infty} \varepsilon^{j} \theta^{j}$, where $\theta^{j}=\theta^{j}(\bar{t}),(\bar{t}=t / \varepsilon)$. Then we find

$$
\sum_{j=0}^{\infty} \varepsilon^{j} \frac{d \theta^{j}}{d \bar{t}}+\sum_{j=0}^{\infty} \varepsilon^{j} \theta(\bar{t})=0
$$

By formal identification at each power of $\varepsilon$, we obtain the following equations:

$$
\frac{d \theta^{j}}{d \bar{t}}+\theta^{j}(\bar{t})=0, \text { for } j \geq 0 .
$$

The initial conditions that we choose are:

$$
\begin{aligned}
& \theta^{0}(0)=\left.u_{0}\right|_{\partial \Omega}-g(0), \\
& \theta^{j}(0)=-k^{j}(0)=(-1)^{j+1} g^{(j)}(0), \text { for } j \geq 1 .
\end{aligned}
$$

By explicit calculations, we obtain:

$$
\theta^{j}=e^{-\frac{t}{\varepsilon}} \theta^{j}(0), \text { for } j \geq 0 .
$$

To obtain the asymptotic error estimate, we set

$$
w_{\varepsilon n}=k^{\varepsilon}-k_{\varepsilon n}-\theta_{\varepsilon n},
$$

where

$$
k_{\varepsilon n}=\sum_{j=0}^{n} \varepsilon^{j} k^{j}, \quad \theta_{\varepsilon n}=\sum_{j=0}^{n} \varepsilon^{j} \theta^{j} .
$$

Now we can conclude as follows. 
Theorem 2.2. If $g^{(n+1)} \in L^{2}\left(0, T ; H^{\frac{1}{2}}(\Gamma)\right)$ for $n \geq 0$, and $w_{\varepsilon n}$ is defined in (2.55), then as $\varepsilon \rightarrow 0$,

$$
\begin{aligned}
& w_{\varepsilon n}=O\left(\varepsilon^{n+1}\right) \text { in } L^{2}\left(0, T ; H^{\frac{1}{2}}(\Gamma)\right), \\
& w_{\varepsilon n}=O\left(\varepsilon^{n+\frac{1}{2}}\right) \text { in } L^{\infty}\left(0, T ; H^{\frac{1}{2}}(\Gamma)\right) .
\end{aligned}
$$

Proof. We first notice that $w_{\varepsilon n}$ vanishes at $t=0$. We then insert (2.55) into (2.2), and we find:

$$
\left\{\begin{array}{l}
\varepsilon\left(w_{\varepsilon n}\right)_{t}+w_{\varepsilon n}=\varepsilon^{n+1}(-1)^{n+1} g^{(n+1)}, \\
\left.w_{\varepsilon n}\right|_{t=0}=0
\end{array}\right.
$$

We take the $H^{\frac{1}{2}}(\Gamma)$ scalar product of $(2.57) 1$ with $w_{\varepsilon n}$ and integrate over $[0, t]$; we obtain

$$
\begin{aligned}
& \frac{\varepsilon}{2}\left|w_{\varepsilon n}(t)\right|_{H^{\frac{1}{2}}(\Gamma)}^{2}+\int_{0}^{t}\left|w_{\varepsilon n}(s)\right|_{H^{\frac{1}{2}}(\Gamma)}^{2} d s=\int_{0}^{t}\left((-\varepsilon)^{n+1} g^{(n+1)}, w_{\varepsilon n}\right)_{H^{\frac{1}{2}}(\Gamma)} d s \\
& \leq \frac{1}{2} \int_{0}^{t}\left|w_{\varepsilon n}(s)\right|_{H^{\frac{1}{2}}(\Gamma)}^{2} d s+\frac{\varepsilon^{2(n+1)}}{2} \int_{0}^{t}\left|g^{(n+1)}(s)\right|_{H^{\frac{1}{2}}(\Gamma)}^{2} d s, \\
& \varepsilon\left|w_{\varepsilon n}(t)\right|_{H^{\frac{1}{2}}(\Gamma)}^{2}+\int_{0}^{t}\left|w_{\varepsilon n}(s)\right|_{H^{\frac{1}{2}}(\Gamma)}^{2} d s \leq \varepsilon^{2(n+1)} \int_{0}^{T}\left|g^{(n+1)}(s)\right|_{H^{\frac{1}{2}}(\Gamma)}^{2} d s .
\end{aligned}
$$

If we set $t=T$ in (2.58), we obtain $w_{\varepsilon n}=O\left(\varepsilon^{n+1}\right)$ in $L^{2}\left(0, T ; H^{\frac{1}{2}}(\Gamma)\right)$, and if we take the supremum of (2.58) over $[0, T]$, we obtain $w_{\varepsilon n}=O\left(\varepsilon^{n+\frac{1}{2}}\right)$ in $L^{\infty}\left(0, T ; H^{\frac{1}{2}}(\Gamma)\right)$.

Theorem 2.2 has been proved.

Remark 2.5. If we additionally assume that $g_{t t} \in L^{2}\left(0, T ; H^{\frac{1}{2}}\right)$ in Theorem 2.1. from (2.56), setting $n=1$, we find $w_{\varepsilon 1}=k^{\epsilon}-g+\epsilon g_{t}-\left(\left.u_{0}\right|_{\partial \Omega}-g(0)\right) e^{-t / \varepsilon}+$ $\varepsilon g_{t}(0) e^{-t / \varepsilon}=O\left(\varepsilon^{3 / 2}\right)$, in $L^{\infty}\left(0, T ; H^{\frac{1}{2}}(\Gamma)\right)$. Then for any $t_{0}>0,\left(k^{\varepsilon}-g\right)\left(t_{0}\right)=$ $-\varepsilon g_{t}\left(t_{0}\right)+O\left(\varepsilon^{3 / 2}\right)+$ e.s.t., where e.s.t. means exponentially small term (for all $H^{m}$-norms).

Hence $\left|\left(k^{\varepsilon}-g\right)\left(t_{0}\right)\right|_{H^{\frac{1}{2}}}=O(\varepsilon)$, for $\forall t_{0}>0$ fixed. We now do similarly as (2.5)-(2.7) for $k_{t}^{\varepsilon}-g_{t}$ and integrate from $t_{0}$ to $t$,

$$
\left|k_{t}^{\varepsilon}-g_{t}\right|_{H^{\frac{1}{2}}(\Gamma)}^{2}(t) \leq e^{-\frac{t-t_{0}}{\varepsilon}}\left|k_{t}^{\varepsilon}-g_{t}\right|_{H^{\frac{1}{2}(\Gamma)}}^{2}\left(t_{0}\right)+\varepsilon\left|g_{t t}\right|_{L^{2}\left(0, T ; H^{\frac{1}{2}}(\Gamma)\right)}^{2},
$$

which yield

$$
k_{t}^{\varepsilon}-g_{t}=O(\sqrt{\varepsilon}) \quad \text { in } \quad L^{\infty}\left(t_{0}, T ; H^{\frac{1}{2}}(\Gamma)\right), \quad \forall t_{0}>0 .
$$

\section{NumericAl RESUlts FOR THE PENALTY METHOD}

3.1. Approximations of $k^{\varepsilon}$. In order to test the efficiency of the proposed penalty method, we will provide in this section and in the next one (Sec. 3.3) some numerical results for system (2.2) with $\Omega=(0,1) \times(0,1)$, and $0 \leq t \leq 1$; we set $g(t)=\sin (t)$ for all $(x, y) \in \partial \Omega, u_{0}(x, y)=\sin \left(\frac{5 \pi}{4} x+\frac{3 \pi}{4}\right) \sin \left(\frac{5 \pi}{4} y+\frac{3 \pi}{4}\right)$, in which case, we face the discrepancies all along the lines $x=0$ and $y=0$ (but no discrepancy along the parts $x=1$ or $y=1$ of the boundary).

We start by testing the quality of the approximation of $k^{\varepsilon}$ inferred by the boundary layer analysis of Section 2.3 that is, $k^{\varepsilon} \sim \sum_{j=0}^{n} \varepsilon^{j}\left(k^{j}+\theta^{j}\right)$, for suitable $\varepsilon^{\prime}$ s and $n$ 's. 

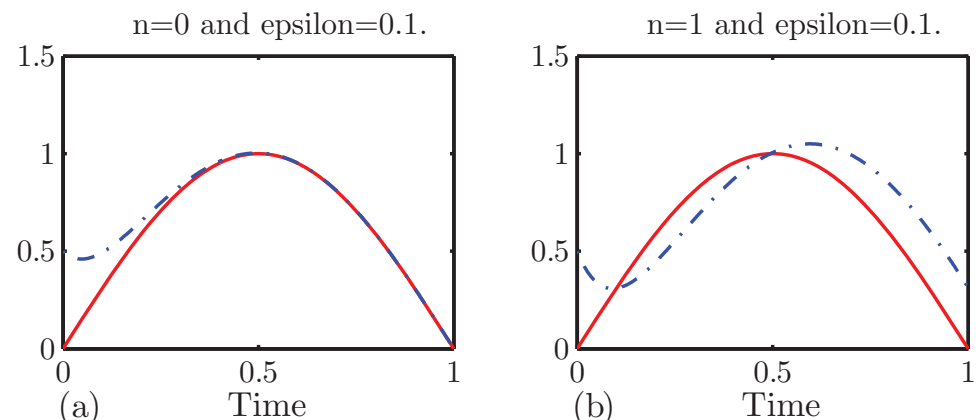

(b)
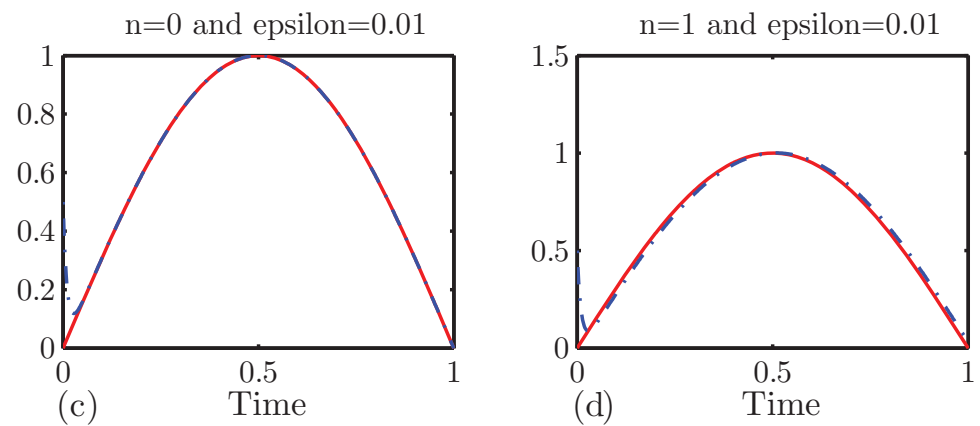

Figure 1. (a) Boundary Layer Element with $\epsilon=0.1, k^{\epsilon} \sim k^{0}+\theta^{0}$,

(b) Boundary Layer Element with $\varepsilon=0.1, k^{\varepsilon} \sim k^{0}+\theta^{0}+\varepsilon k^{1}+\varepsilon \theta^{1}$,

(c) Boundary Layer Element with $\varepsilon=0.01, k^{\varepsilon} \sim k^{0}+\theta^{0}$, (d)

Boundary Layer Element with $\varepsilon=0.01, k^{\varepsilon} \sim k^{0}+\theta^{0}+\varepsilon k^{1}+\varepsilon \theta^{1}$.

Because (2.2) is a $2 D$ system which makes the graphing impossible along the time axis, we restrict ourselves to follow the time evolution of the exact and approximate function at one point of the boundary; for simplicity we choose the corner $(x, y)=$ $(0,0)$. We then plot in Figure 1, $g(t)$ (solid line) and $k^{\varepsilon}$ (dash-dot line), $k^{\varepsilon} \sim$ $\sum_{j=0}^{n} \varepsilon^{j}\left(k^{j}+\theta^{j}\right)$ with $n=0$ or 1 , and $\varepsilon=0.1$ or 0.01 . For $n=1$, the proposed new scheme gives a good approximation of $k^{\varepsilon}$.

Figure 2 gives the $L^{2}-$ and $L^{\infty}-$ errors which stand, respectively, for the $L^{2}\left(0, T ; H^{\frac{1}{2}}(\Gamma)\right)$ and $L^{\infty}\left(0, T ; H^{\frac{1}{2}}(\Gamma)\right)$ norms of the difference between the real solution $k^{\varepsilon}$ and the approximations $\sum_{j=0}^{n} \varepsilon^{j}\left(k^{j}+\theta^{j}\right)$, as the number of time steps $T$, $\varepsilon$ and $n$ vary. It is clear that the smaller $\varepsilon$ is, the smaller both errors are.

3.2. A two-dimensional system in a square $\Omega$. To verify the effectiveness of the penalty method, we use the finite elements method for the spatial approximation of $u$. The Penalty Method is mainly aimed for multi-dimensional time-dependent PDEs, so we consider the $2 D$ system, as in (1.2):

$$
\left\{\begin{array}{l}
\frac{\partial u}{\partial t}-\nu\left(u_{x x}+u_{y y}\right)=f, \\
\left.u\right|_{\partial \Omega}=g \\
\left.u\right|_{t=0}=u_{0} .
\end{array}\right.
$$

where $0 \leq x \leq 1,0 \leq y \leq 1,0 \leq t \leq 1$ 

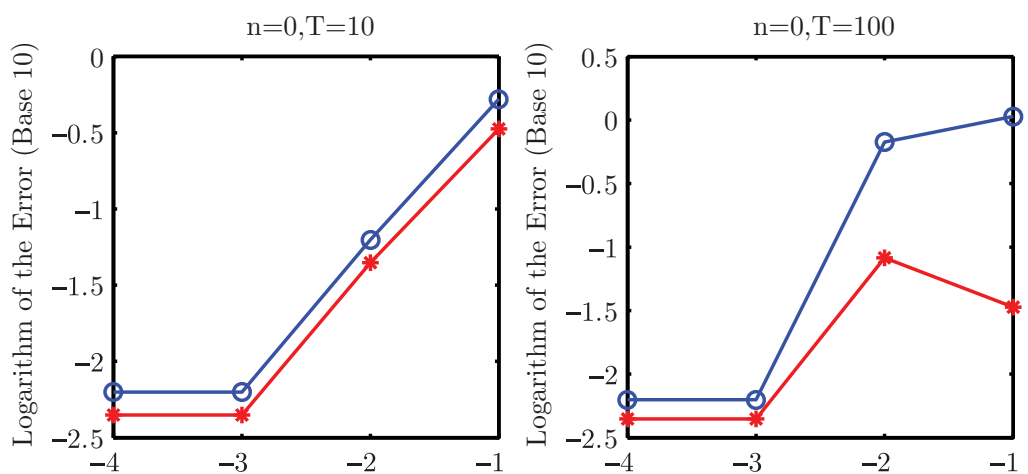

(a) Logarithm of Epsilon (Base 10)

(b) Logarithm of Epsilon (Base 10)

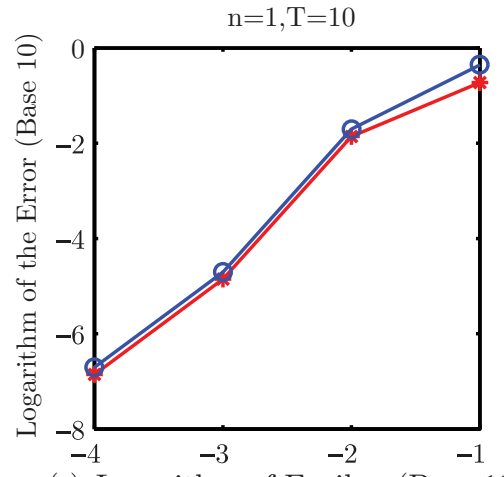

(c) Logarithm of Epsilon (Base 10)

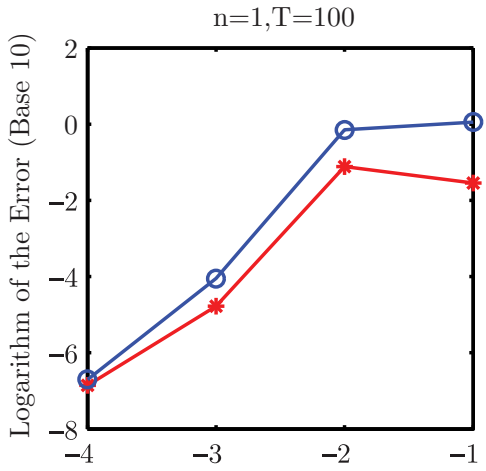

(d) Logarithm of Epsilon (Base 10)

Figure 2. $L^{2}$ (plot o)- and $L^{\infty}$ (plot $*$ )- error between the boundary layer schemes and the real solution for $\varepsilon k_{t}+k=g$.

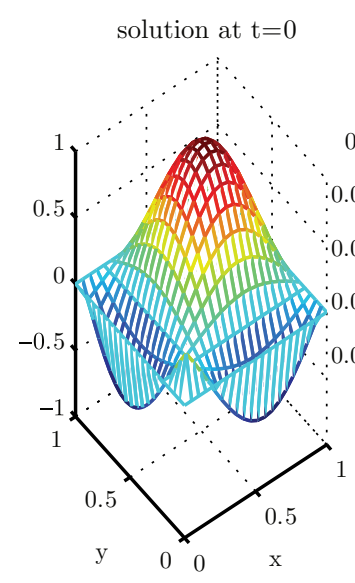

(a)

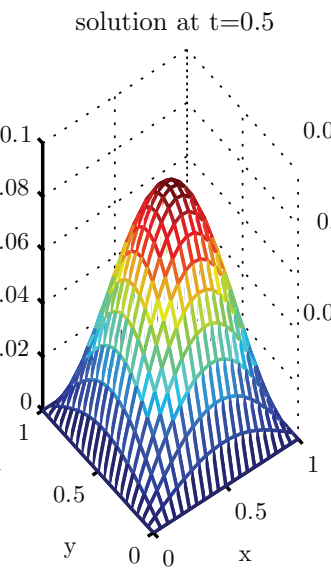

(b)



(c)

Figure 3 . The exact solution of the system in the square $\Omega$ without applying the penalty method, at times $0,0.5$ and 1 (Figures [3(a), 3 (b), 迆(c)). 

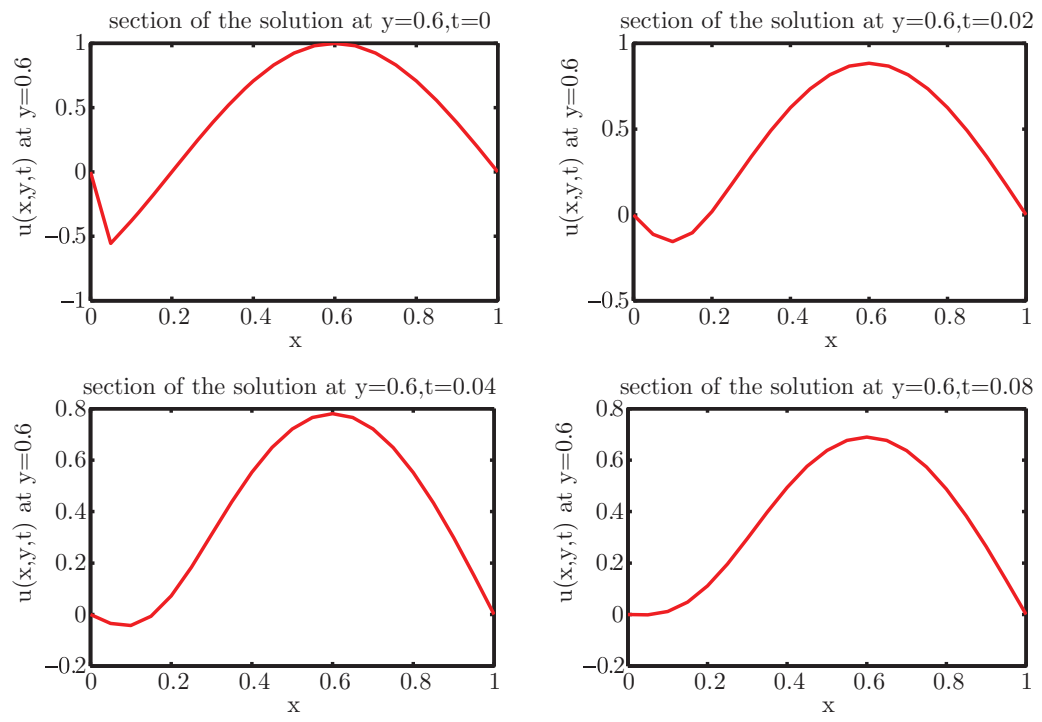

Figure 4 . The sections of the exact solution at $y=0.6$ when $t$ is close to 0 .

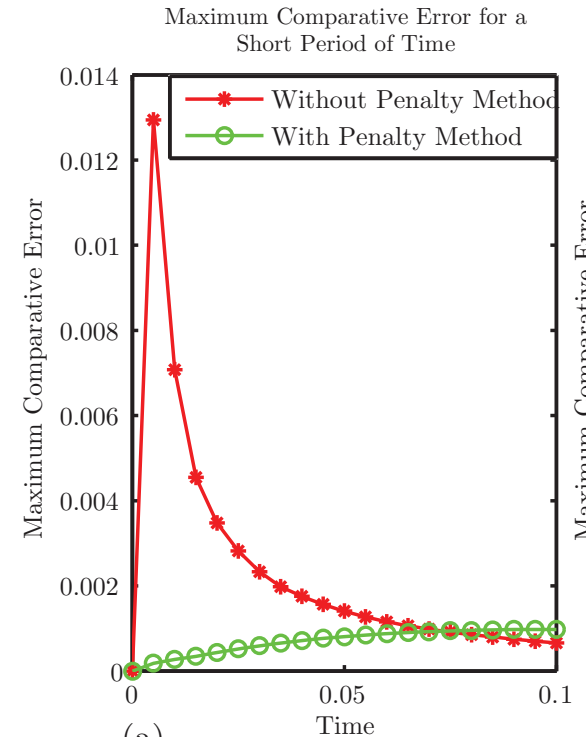

(a)

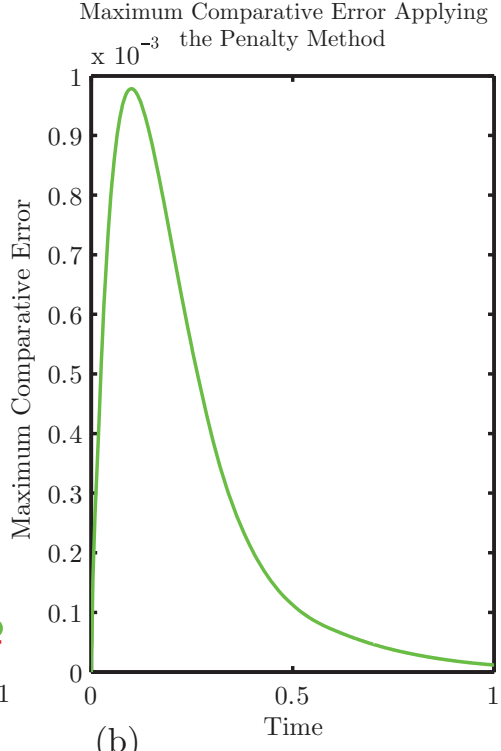

(b)

Figure 5. The comparative errors of the $2 \mathrm{D}$ system in the $L^{\infty}$ norm for $\varepsilon=0.1$. (a) Maximum comparative error for a short time period (in real value), (b) Maximum comparative error when applying the Penalty Method (times $10^{-3}$ ). 

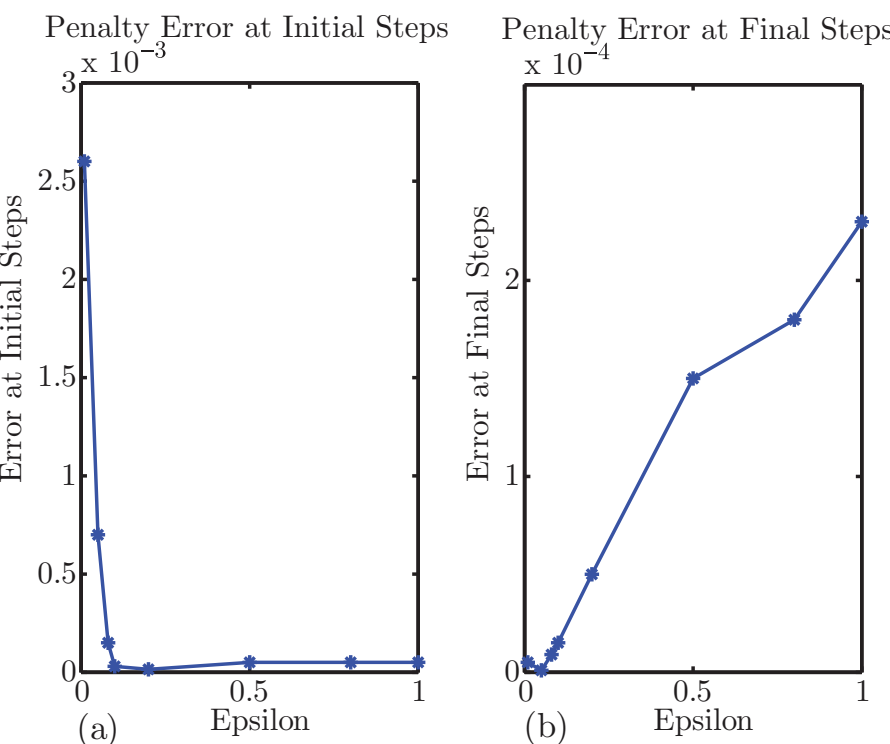

Figure 6 . The comparative errors for the $2 \mathrm{D}$ system in the $L^{\infty}$ norm with variations of $\epsilon$ with mesh $\triangle x=\frac{1}{24}, \triangle y=\frac{1}{24}, \triangle t=\frac{1}{1000}$. (a) The error at initial step. (b) The error at final step. Note the factors $10^{-3}, 10^{-4}$ in (a), (b)

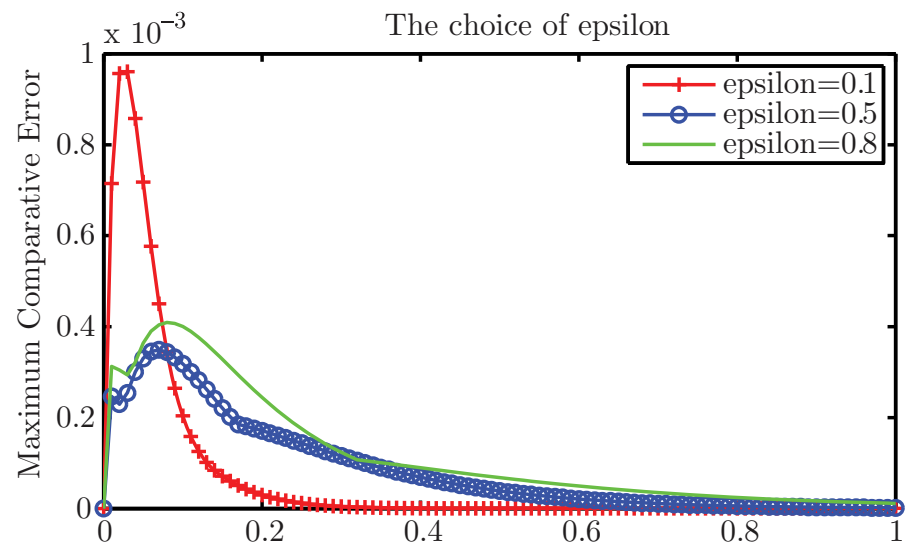

FiguRE 7 . The maximum comparative errors for the $2 \mathrm{D}$ system in square domain. 


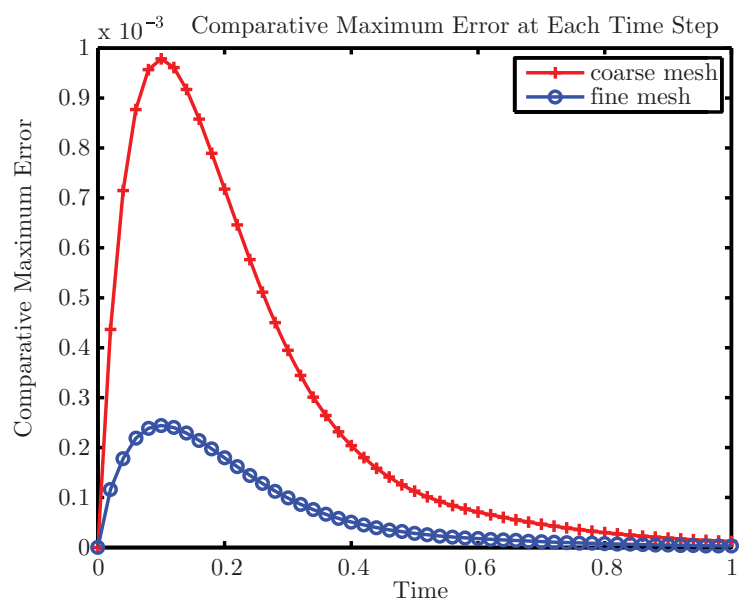

Figure 8. The comparative errors for the $2 \mathrm{D}$ system in $L^{\infty}$ sense at $\epsilon=0.1$. The upper line is with mesh $\triangle x=\frac{1}{24}, \triangle y=\frac{1}{24}, \triangle t=$ $\frac{1}{1000}$, the lower line is with mesh $\triangle x=\frac{1}{48}, \triangle y=\frac{1}{48}, \triangle t=\frac{1}{4000}$
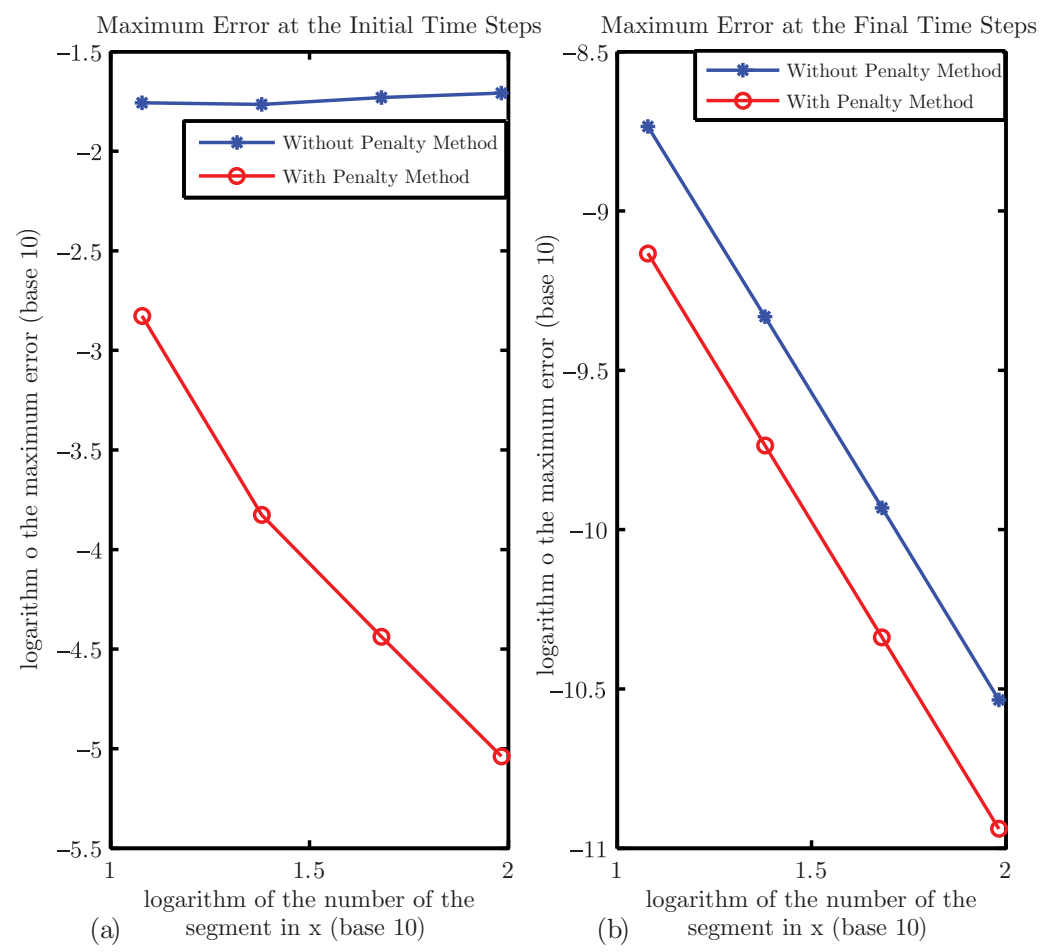

Figure 9. Decay of the maximum errors. When we apply the penalty method here $\varepsilon=0.1$. (a) at the initial steps, (b) at the final steps 
We set $\nu=0.2, f=0, g=0, u_{0}=\sin \left(\frac{5 \pi}{4} x+\frac{3 \pi}{4}\right) \sin \left(\frac{5 \pi}{4} y+\frac{3 \pi}{4}\right)$, so that $g(0) \neq\left. u_{0}\right|_{\partial \Omega}$ on the lines $x=0$ and $y=0$. So we face the incompatibility problem, namely the boundary conditions and the initial condition do not match at these corners of the time and spatial axes. For the test we set $\epsilon=0.1$ in the penalty approximation (2.1) - (2.2) of (3.1). In more general problems, we might have discontinuities at the space corners $x=0$ or $1, y=0$ or 1 . But since the function $u_{0}$ is smooth at the corners, these singularities do not occur here, at least at the low orders.

We first plot the solution of system (3.1) without applying the penalty method. The solution is plotted in Figure 3. (a) is the graph of the approximate solution at $t=0,(\mathrm{~b})$ is the graph of the approximate solution at $t=0.5$, and (c) is the graph of the approximate solution at $t=1$. The graph displays a sharp gradient around the corner of the time-space axis during an initial short period due to the incompatibility between the initial and boundary conditions there. In order to see the sharp gradient clearly and the changes of the gradient as time evolves, we plot the sections $(x \in(0,1), y=0.6)$ of the solution at times close to 0 ; see Figure 4 It is clear that, at $t=0$, we observe the sharpest gradient at the time-space corner and as time evolves, the gradient becomes smoother and smoother at that corner, until $t=0.08$, when it is essentially flat.

Next, to study the accuracy of the numerical method, we must measure the errors for the approximate solutions. Hence we compute the comparative errors which are the differences between two numerical solutions for the problem, one with the stated mesh sizes, and the other one with a finer mesh. Then at each time step, we obtain the maximum error between the two meshes above; it is understood to be $L^{\infty}$ comparative errors, or maximum comparative errors. In what follows, all the error terms are to be understood in this sense.

We plot the maximum comparative errors of the $2 D$ system on Figure 5 , Graph (b) is the plot of the maximum comparative errors along the whole time period if we apply the penalty method. Because the discrepancy happens at the time-space corner, we zoom into the left corner of graph (b) and compare it with the error when we do no apply the penalty method. In graph (a), the line with stars is the maximum comparative errors with the penalty method applied, and the line with circles is the maximum comparative errors without the penalty method. We observe that the magnitude of the errors at the time-space corner is reduced by around one order by the penalty method.

Because we use finite difference methods, for the same $\varepsilon$, if we have a finer mesh, the maximum comparative error should be smaller. In Figure 8 we plot the error of the $2 D$ system with $\varepsilon=0.1$, the lower curve with a finer mesh, the upper curve with a coarser mesh. The magnitude of the errors is reduced by around $40 \%$. So for a fixed $\varepsilon$, the finer the mesh is, the smaller the error is. We are also interested in the decay of the maximum errors. The most interesting and informative comparison can be made between the decay rates of the maximum errors at the initial and final time steps. In Figure 9 (a), the maximum errors at the initial time step are plotted against the grid resolution in the log-log scale. Without the penalty method, the maximum errors do not decrease as the grid refines, which demonstrates that the singularity in the solution during the initial period is serious. With the penalty method $(\varepsilon=0.1)$, the maximum errors decay at roughly the second order. Figure 
9 (b) shows that, with and without applying penalty method, the maximum errors at the final steps $(t=1)$ decay at approximately the second order.

Next, we fix the meshes, e.g., at $\triangle x=\frac{1}{24}, \triangle y=\frac{1}{24}, \triangle t=\frac{1}{1000}$, and let $\varepsilon$ vary. In Figure 6, we plot the maximum comparative errors of system (3.1). At the initial steps, the error decreases sharply as $\varepsilon$ increases and remains close to 0 , then it becomes stable flat. At the final steps, the error increases almost linearly as $\varepsilon$ increases. With $\varepsilon$ at about 0.1 , the initial error is minimized while the error at final step is well controlled. But as Figure 7 shows, in a short time period $\varepsilon=0.5$ gives us smaller errors and again after a short time period $\varepsilon=0.1$ gives us a smaller error. In optimization theory, the choice of $\varepsilon$ is usually made by trial and error and is not a major issue. It does not follow the "intuitive" idea that the error becomes smaller as $\varepsilon$ becomes smaller because of many other contingent errors such as round-off and descretization errors. In general the choice of $\varepsilon$ depends on our goals of the computation.

3.3. 2D system in a disk $\Omega$. To further verify the effectiveness of the penalty method we now test the results in a different domain. We now choose a disk $\Omega=\left\{(x, y) \mid x^{2}+y^{2} \leq 1\right\}$. The $2 \mathrm{D}$ heat equations in the polar coordinates $x=$ $r \cos (\theta), y=r \sin (\theta)$ where $0 \leq \theta \leq 2 \pi, 0 \leq r \leq 1 \mathrm{read}$

$$
\left\{\begin{array}{l}
\frac{\partial u}{\partial t}-\nu\left(u_{r r}+\frac{u_{r}}{r}+\frac{u_{\theta \theta}}{r^{2}}\right)=f, \\
\left.u\right|_{t=0}=u_{0}, \\
\left.u\right|_{r=1}=g .
\end{array}\right.
$$

Consider the $2 D$ system (3.2), where $0 \leq t \leq 1$, and set $\nu=0.2, f=0, g=0$ and $u_{0}(x, y)=x y$, so that $g(0) \neq\left. u_{0}\right|_{\partial \Omega}$. We also set $\varepsilon=0.1$ the same as before. In this case, we face the singularities almost everywhere along the unit circle except at

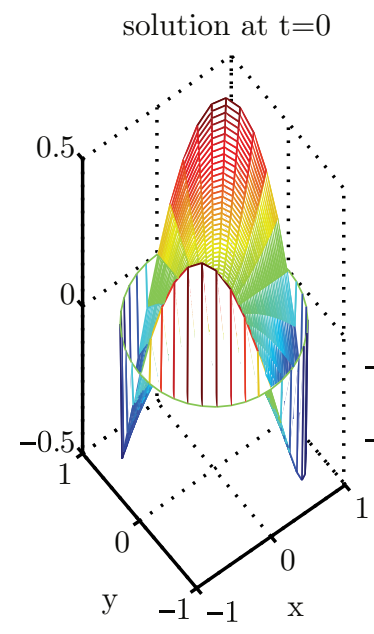

(a)

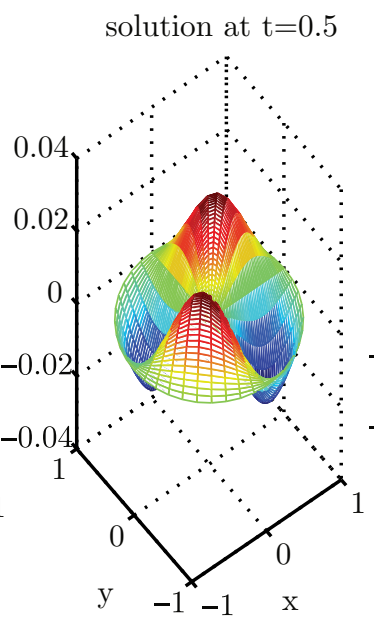

(b)

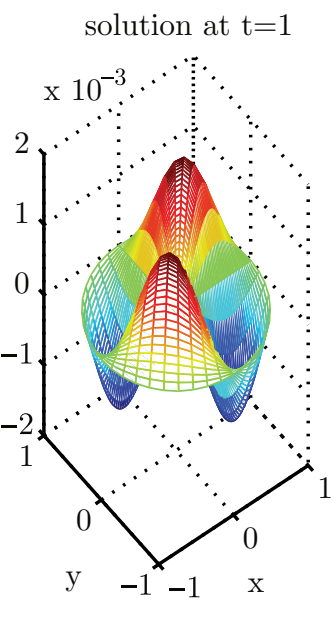

(c)

Figure 10. The solution of the system in disk $\Omega$ without applying the penalty method. 

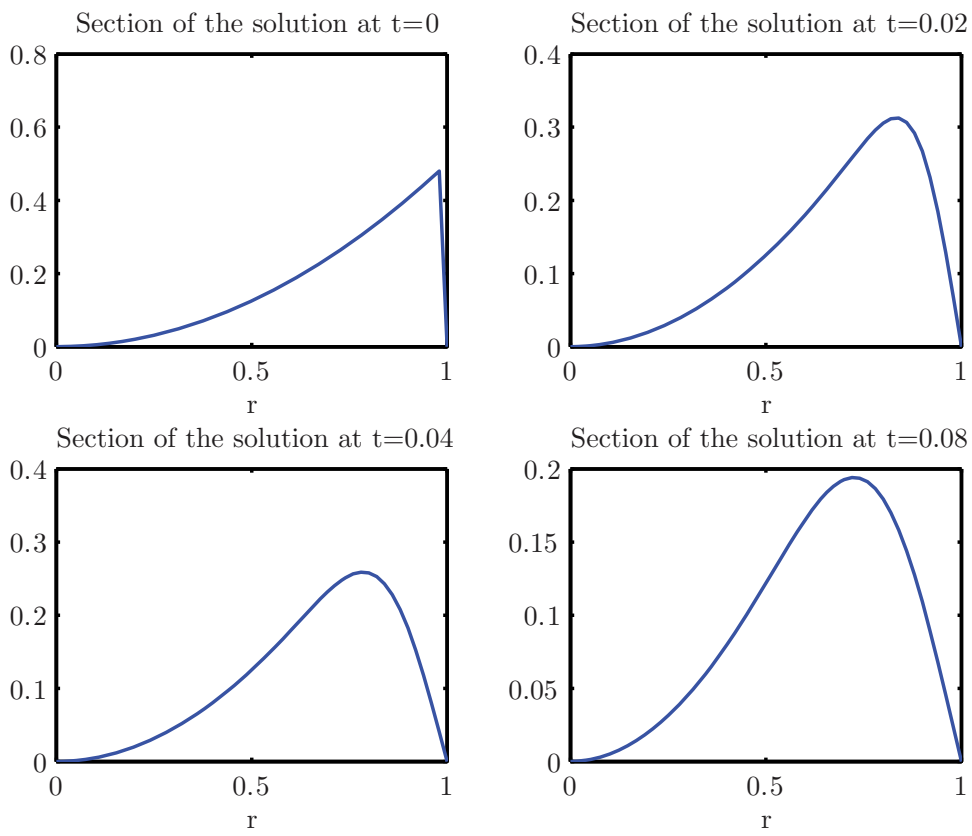

Figure 11. The section of the solution at $\theta=\frac{\pi}{4}$ when $t$ is close to 0 .
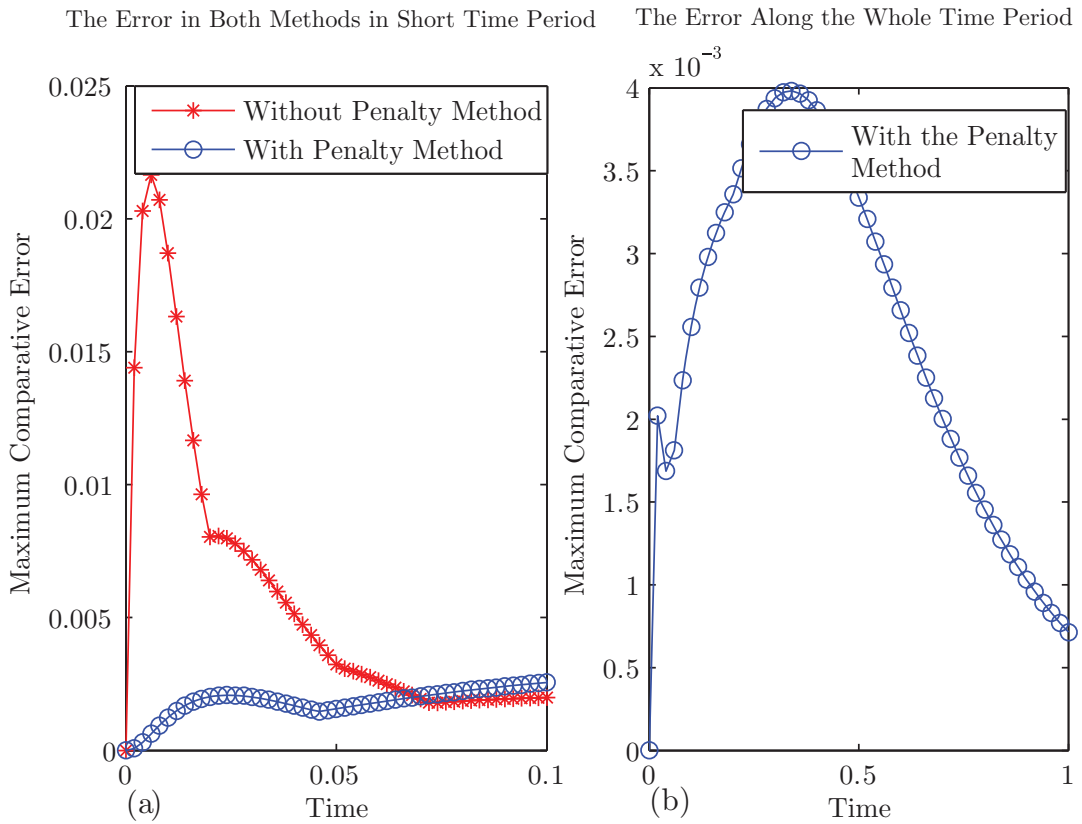

FiguRe 12. The maximum comparative error for Ordinary Finite Element and penalty method in $L^{\infty}$ sense with $\varepsilon=0.1$ 


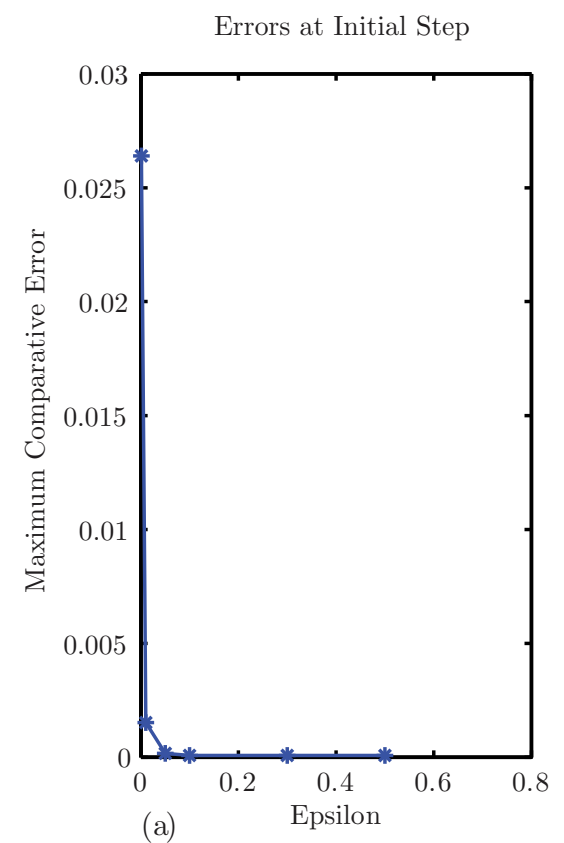

(a)

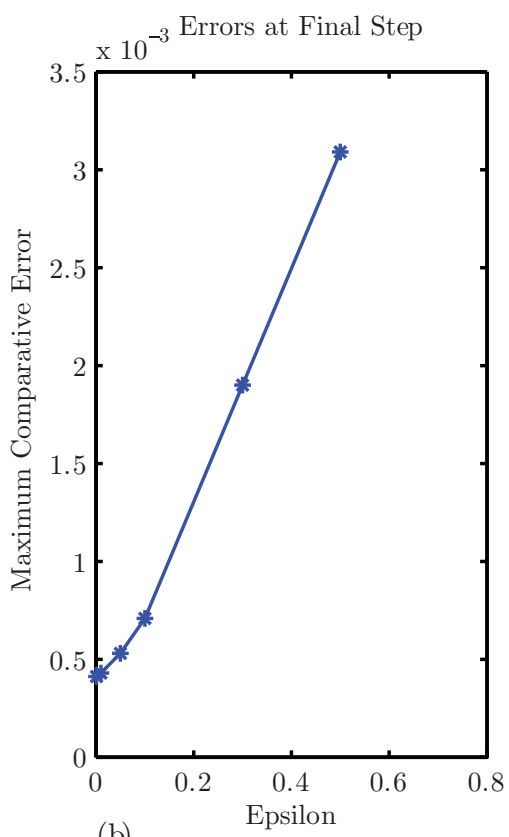

(b)

FIGURE 13. The maximum comparative errors for penalty method at both initial and final steps as $\varepsilon$ variants with mesh $\triangle r=$ $\frac{1}{10}, \triangle \theta=\frac{1}{10}, \triangle T=\frac{1}{1000}$.

the points where $x=0$ or $y=0$. The effectiveness of this method will be verified with the following numerical results.

We first compute the solution of (3.2) without applying the penalty method. The solution is plotted in Figure 10, (a) is the graph of the solution at $t=0$, (b) is the graph of the solution at $t=0.5$, and (c) is the graph of the solution at $t=1$. As we did for the system (3.1) for the square, we plot in Figure 11 the sections $\left(r \in(0,1), \theta=\frac{\pi}{4}\right)$ of the solution at times close to 0 . It is clear that, at $t=0$, the graph displays a sharp gradient around the corner of the time-space axis due to the discrepancy between the initial and boundary conditions there, and as time evolves, the gradient becomes smoother and smoother.

To study the error of the system in the disk $\Omega$, we define the maximum comparative errors as for the square $\Omega$. Hence we plot the $L^{\infty}$ errors for the $2 D$ system for the disk $\Omega$ on Figure 12 graph (b) is the maximum comparative error along the whole time period if we apply the penalty method. Because the discrepancy happens at the time-space corner, we zoom into the left corner of graph (b) and compare it with the error when we do not apply the penalty method. From graph (a), we observe that the magnitude of the errors at the time-space corner are reduced by a factor of 10 if we apply the penalty method.

In Figure 13, we plot the maximum comparative error for (3.2) with a fixed mesh at both initial and final steps. At the initial step, the error decreases sharply as $\varepsilon$ increases and remains close to 0 , and then it becomes flat. At the final step, the error increases almost linearly as $\epsilon$ increases. The observation also leads to the 

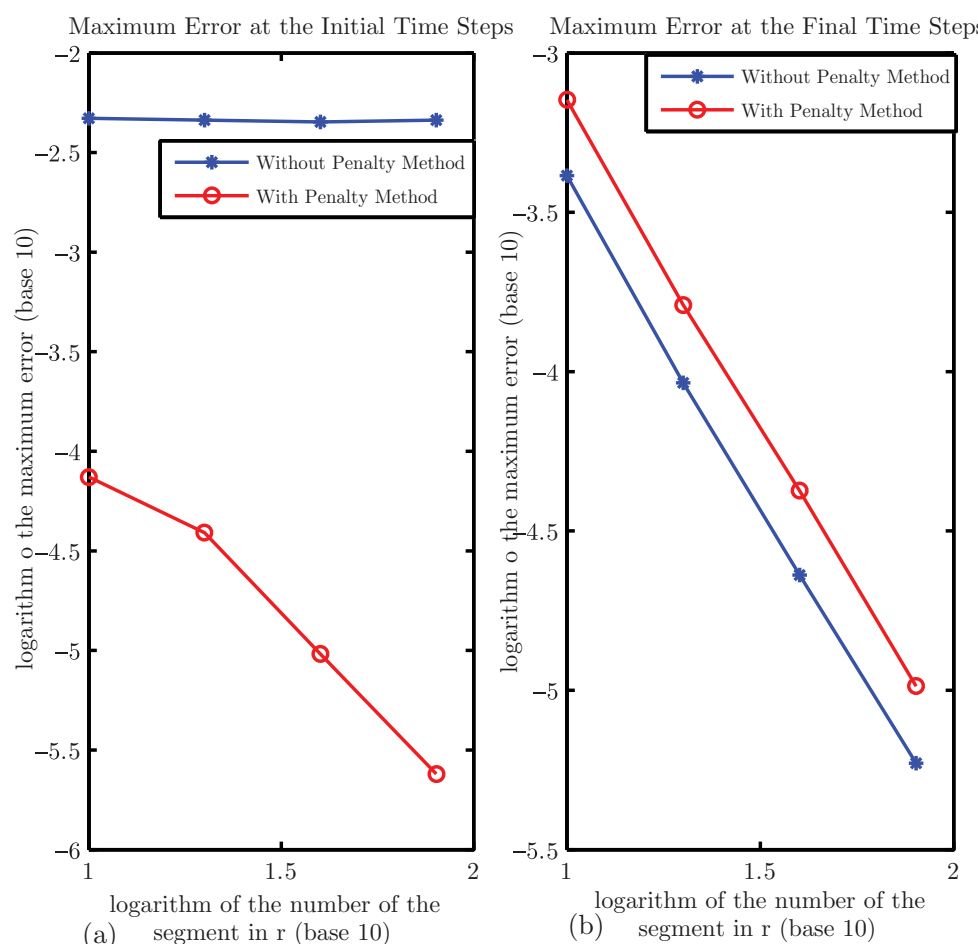

Figure 14. Decay of the maximum errors. When we apply the penalty method here $\varepsilon=0.1$. (a) at the initial steps, (b) at the final steps

following conclusion: at about $\varepsilon=0.1$, the initial error is minimized while the error at final step is well controlled.

As for the previous example, we shall now look at how the singularity, induced by the compatibility between the initial and boundary data, affects the convergence rates of the numerical scheme. In Figure 14 we plot the maximum errors, at the initial and final time steps, with and without the penalty method, against the spatial resolution in the log-log scale. We see in Figure 14 (a) that, without the penalty method, the maximum errors do not decrease as the grid refines, which demonstrates that the singularity in the solution at the initial time step is serious. With the penalty method, the maximum errors decay at roughly the second order. Figure14(b) shows that, with and without applying penalty method, the maximum errors at the final steps $(\mathrm{t}=1)$ decay at approximately the second order.

3.4. Implementation in a $1 \mathrm{D}$ system. As we said in the Introduction the penalty method applies without any restriction on space dimension. However, a number of methods have previously been proposed which only apply to space dimension one. Our aim is now to compare the efficiency of the penalty method with some of the earlier methods; and therefore we can only consider the case of space dimension 1. More precisely, we will consider the Corrector Methods as proposed in $[8-10$ and compare them with the penalty method for the $1 D$ system 


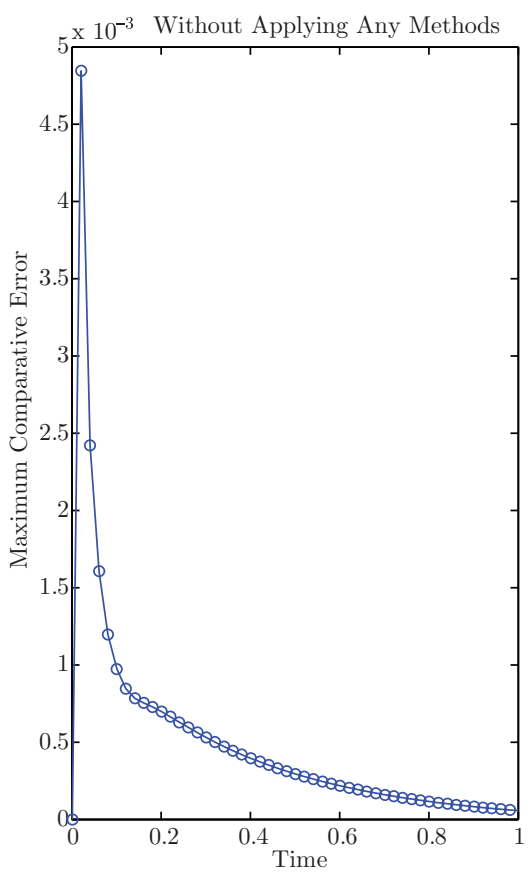

(a)

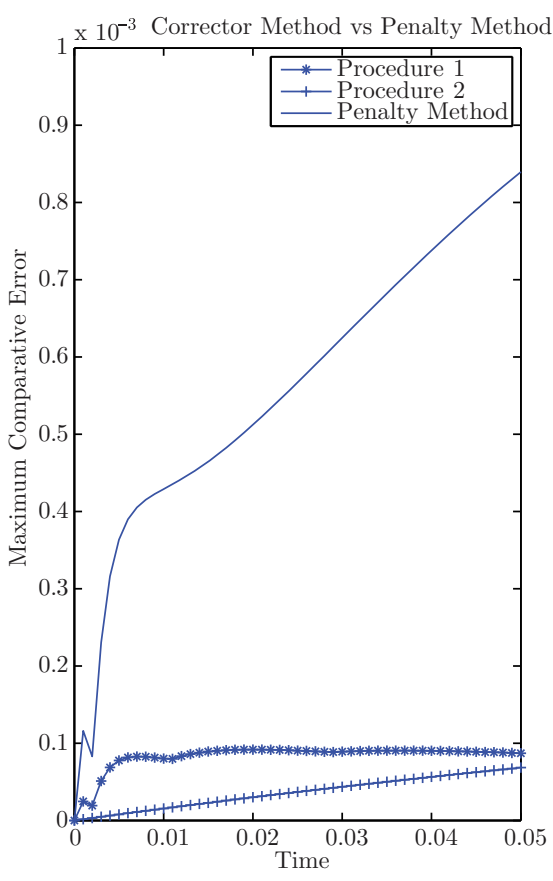

(b)

FiguRE 15. Comparative error of the two methods in 1D system in $L^{\infty}$ sense at $\varepsilon=0.1$

$$
\left\{\begin{array}{l}
u_{t}-\nu u_{x x}=0, \quad 0<x<1, \quad 0<t<1, \\
u(x, 0)=u_{0}, \\
u(0, t)=g_{1}(t), \quad u(1, t)=g_{2}(t) .
\end{array}\right.
$$

Here we set $u_{0}(x)=\sin \left(\frac{5 \pi}{4} x+\frac{3 \pi}{4}\right), g_{1}(t)=0, g_{2}(t)=0, \nu=0.2$. For the Penalty Method, we also set $\varepsilon=0.1$, and for the Corrector Method, we have the following choice of correctors [5]-9] offering increasing accuracy:

$$
S=\left\{\begin{array}{lr}
0, & \text { (Procedure 1), } \\
\alpha_{0} S_{0}, & \text { (Procedure 2), }
\end{array}\right.
$$

where $\alpha_{0}=g_{1}(0)-u_{0}(0), \alpha_{1}=g_{1 t}(0)-u_{0 x x}(0), S_{0}=\frac{1}{\sqrt{\pi \nu t}} \int_{x}^{\infty} e^{-\frac{s^{2}}{4 \nu t}} d s=$ $\operatorname{erfc}\left(\frac{x}{\sqrt{\nu t}}\right)$ and $S_{1}=\int_{0}^{t} S_{0}(x, \tau) d \tau$. Here Procedure 1 absorbs the $0^{t h}$ order incompatibility $\left(g_{1}(0) \neq u_{0}(0)\right)$, and Procedure 2 absorbs both the $0^{\text {th }}$ and $1^{\text {st }}$ order incompatibilities $\left(g_{1}(0) \neq u_{0}(0)\right.$ and $\left.g_{1 t}(0) \neq \nu u_{0 x x}(0)\right)$.

Let $u=v+S$; we see that $v$ is the solution of the following equation:

$$
\left\{\begin{array}{l}
v_{t}-\nu v_{x x}=0,0<x<1,0<t<1, \\
v(x, 0)=u_{0}(x), \\
v(0, t)=g_{1}(t)-S(0, t), \quad v(1, t)=g_{2}(t)-S(1, t) .
\end{array}\right.
$$




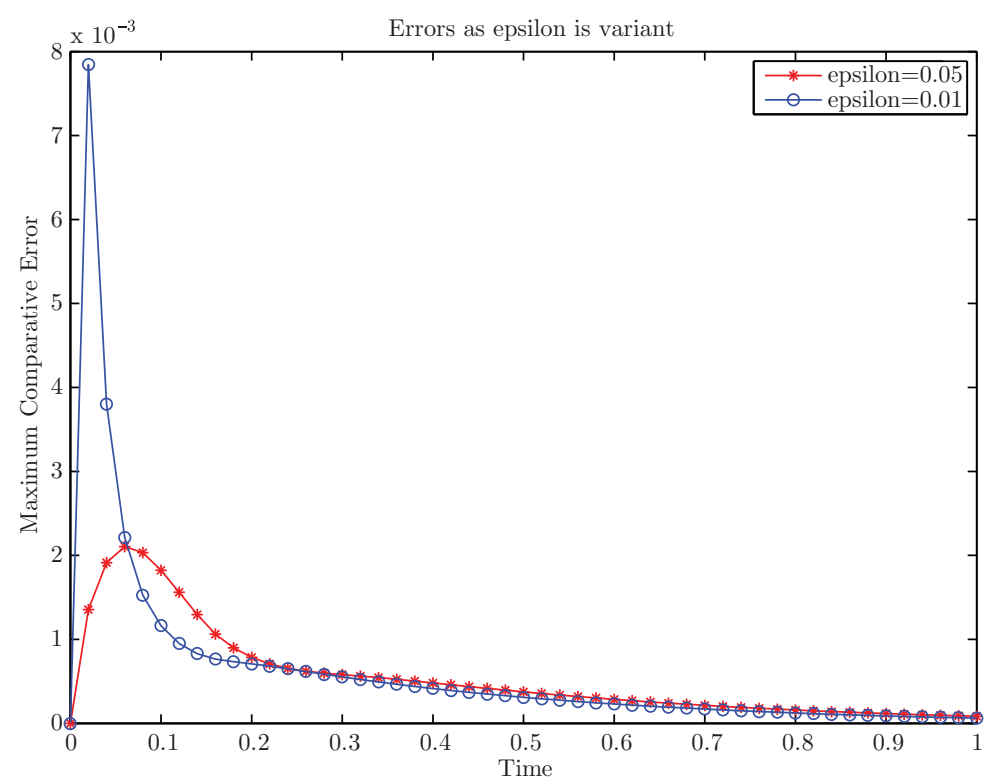

Figure 16. The maximum comparative errors for the 1D system in $L^{\infty}$ sense along the time

We choose to solve equation (3.5) by finite differences. Figure 15 gives the comparison between different methods (Penalty Method and Correction Method). Figure 15 (a) gives us the maximum comparative error of system (3.3) without applying any methods. Figure 15 (b) compares the two methods, zooming into the corner of the time-space domain where errors are the largest due to the incompatibility at $t=0$. As expected Procedure 2 gives slightly better results than Procedure 1. Also, the errors with the penalty method are larger than with both procedures, but still of comparable magnitude whereas the errors without any procedure reach a pick about 6 times larger $\left(4.8 \times 10^{-3}\right.$ vs $\left.0.8 \times 10^{-3}\right)$. Now we want to vary $\varepsilon$ in this 1D system, Figure 16 shows that if $\varepsilon$ is too small as compared to the mesh, the Penalty Method would not reduce the errors at the spatio-temporal corner, but if it is an appropriate small number, it could really reduce the errors by more than $80 \%$.

\section{Conclusion}

The penalty method gives a way to solve the higher dimensional incompatibility problems. As expected, there exists a solution for system (1.2) which is continuous over $\left[t_{0}, T\right]$, for all $t_{0}>0$.

The discrepancy occurs at the time-space corner; we are effectively interested in the errors for the initial short time period. The numerical simulations for the system with both a square $\Omega$ and a disk $\Omega$ yield similar results. At the spatiotemporal corner, the magnitudes of the errors are reduced by about one order of magnitude by the penalty method. Tests are also conducted to study the effects of different values of $\varepsilon$, the key parameter in the penalty method. We find that with 
an appropriate small value for $\varepsilon$, the initial error can be minimized while the error at final step is under well controlled.

Finally, in space dimension one, when both methods are available (penalty method and correction procedures 1 and 2), the penalty method gives a slightly larger error than the Correction Procedures 1 and 2; but the order of magnitude of the errors are comparable and they are all significantly smaller than the errors appearing when no correction procedure is implemented .

\section{Appendix: The USER GUIDE}

The aim is to address the incompatibility issue for the multi-dimensional timedependent linear parabolic equation

$$
\left\{\begin{array}{l}
u_{t}-\nu \triangle u=f, \quad x \in \Omega \subset R^{d}, \quad t \in R^{+}, \\
\left.u\right|_{t=0}=u_{0}, \\
\left.u\right|_{\partial \Omega}=g
\end{array}\right.
$$

where $\left.u_{0}\right|_{\partial \Omega} \neq\left. g\right|_{t=0}$. So we consider new system instead, namely, for $\varepsilon>0$ fixed,

$$
\begin{aligned}
& \left\{\begin{array}{l}
u_{t}^{\varepsilon}-\nu \triangle u^{\varepsilon}=f, \quad x \in \Omega \subset R^{d}, \quad t \in R^{+}, \\
\left.u^{\varepsilon}\right|_{t=0}=u_{0}, \\
\left.u^{\varepsilon}\right|_{\partial \Omega}=k^{\varepsilon},
\end{array}\right. \\
& \left\{\begin{array}{l}
k_{t}^{\varepsilon}+\frac{1}{\varepsilon}\left(k^{\varepsilon}-g\right)=0, \quad t \in R^{+}, \\
k^{\varepsilon}(0)=\left.u_{0}\right|_{\partial \Omega} .
\end{array}\right.
\end{aligned}
$$

We consider, for instance, the rectangle $0 \leq x \leq 1,0 \leq y \leq 1$ and $0 \leq t \leq 1$. We consider the discretization meshes $\triangle x=1 / M, \triangle y=1 / N$ and $\triangle t=1 / T$, where $M, N, T$ are integers. We use an explicit scheme to compute the numerical solution of the original system (4.1) and of the modified system (4.2), (4.3), that is, respectively:

$$
\left\{\begin{array}{l}
\frac{u_{i, j}^{n+1}-u_{i, j}^{n}}{\triangle t}-\nu\left(\frac{u_{i+1, j}^{n}+u_{i-1, j}^{n}-2 u_{i, j}^{n}}{\triangle x^{2}}+\frac{u_{i, j+1}^{n}+u_{i, j-1}^{n}-2 u_{i, j}^{n}}{\triangle y^{2}}\right)=f_{i, j}^{n} \\
\text { for } 1 \leq i \leq N-1, \quad 1 \leq j \leq M-1, \quad 1 \leq n \leq T \\
\left.u_{i, j}^{n}\right|_{\partial \Omega}=\left.g_{i, j}(n \triangle t)\right|_{\partial \Omega}, \text { for } i=0, N \text { or } j=0, M \\
u_{i, j}^{0}=u_{0}(i \triangle x, j \triangle y), \text { for } 0 \leq i \leq N, \quad 0 \leq j \leq M
\end{array}\right.
$$

for (4.1), and, for (4.2)-(4.3):

$$
\left\{\begin{array}{l}
\frac{u_{i, j}^{n+1}-u_{i, j}^{n}}{\triangle t}-\nu\left(\frac{u_{i+1, j}^{n}+u_{i-1, j}^{n}-2 u_{i, j}^{n}}{\triangle x^{2}}+\frac{u_{i, j+1}^{n}+u_{i, j-1}^{n}-2 u_{i, j}^{n}}{\triangle y^{2}}\right)=f_{i, j}^{n}, \\
\text { for } 1 \leq i \leq N-1, \quad 1 \leq j \leq M-1, \quad 1 \leq n \leq T, \\
\left.u_{i, j}^{n}\right|_{\partial \Omega}=\left.k_{i, j}^{\varepsilon n}\right|_{\partial \Omega}, \text { for } i=0, N \text { or } j=0, M \\
u_{i, j}^{0}=u_{0}(i \triangle x, j \triangle y), \text { for } 0 \leq i \leq N, 0 \leq j \leq M \\
\frac{k_{i, j}^{\varepsilon n+1}-k_{i, j}^{\varepsilon n}}{\triangle t}+\frac{1}{\varepsilon}\left(k_{i, j}^{\varepsilon n}-g_{i, j}(n \triangle t)\right)=0 \\
\text { for } i=0, N \text { or } j=0, M, n \geq 1, \\
k_{i, j}^{\varepsilon 0}=u_{0}(i \triangle x, j \triangle y), \text { for } i=0, N \text { or } j=0, M
\end{array}\right.
$$




\section{ACKNOWLEDGMENTS}

This work was partially supported by the National Science Foundation under the grants NSF-DMS-0604235, and DMS-0906440 and by the Research Fund of Indiana University.

\section{REFERENCES}

1. L.K. Bieniasz, A singularity correction procedure for digital simulation of potential-step chronoamperometric transients in one-dimensional homogeneous reaction-diffusion systems, Electrochimica Acta 50 (2005), 3253-3261.

2. John P. Boyd and Natasha Flyer, Compatibility conditions for time-dependent partial differential equations and the rate of convergence of Chebyshev and Fourier spectral methods, Comput. Methods Appl. Mech. Engrg. 175 (1999), no. 3-4, 281-309. MR.1702205 (2000d:65183)

3. J.P. Boyd and N. Flyer, Compatibility conditions for time-dependent partial differential equations and the rate of convergence of Chebyshev and Fourier spectral methods (english. english summary), Methods Appl. Mech. Eng. 175(3-4) (1999), 281-309. MR1702205(2000d:65183)

4. John Rozier Cannon, The one-dimensional heat equation, Encyclopedia of Mathematics and its Applications 23 (1984), xxv+483 pp. MR747979 (86b:35073)

5. Qingshan Chen, Zhen Qin, and Roger Temam, Numerical resolution near $t=0$ of nonlinear evolution equations in the presence of corner singularities in space dimension 1, Commun. Comput. Phys. 9 (2011), no. 3, 568-586. MR2726818

6. R. Courant, Variational methods for the solution of problems of equilibrium and vibrations, Bull. Amer. Math. Soc. 49 (1943), 1-23. MR0007838 (4:200e)

7. M.S. Engelman, R.L. Sani, and P.M. Gresho, The implementation of normal and/or tangential boundary conditions in finite element codes for incompresible fluid flow, Int. J. Numer. Methods Fluids 2(3) (1982), 225-238,76-08. MR667793 (83g:76014)

8. Natasha Flyer and Bengt Fornberg, Accurate numerical resolution of transients in initialboundary value problems for the heat equation, J. Comput. Phys. 184 (2003), no. 2, 526-539. MR:1959406 (2003m:65154)

9. __ On the nature of initial-boundary value solutions for dispersive equations, SIAM J. Appl. Math. 64 (2003/04), no. 2, 546-564 (electronic). MR.2049663 (2005a:35006)

10. Natasha Flyer and Paul N. Swarztrauber, The convergence of spectral and finite difference methods for initial-boundary value problems, SIAM J. Sci. Comput. 23 (2002), no. 5, 17311751 (electronic). MR1885081 (2002k:65122)

11. R. Foias, C.; Temam, Some analytic and geometric properties of the solutions of the evolution navier-stokes equations, J. Math. Pures Appl. 9 (1979), no. 3, 339-368. MR544257 (81k:35130)

12. P.M. Gresho, Incompressible fluid-dynamics - some fundamental formulation issues, Annu. Rev. Fluid Mech. 23 (1991), 413-453. MR.1090333 (92e:76017)

13. P.M. Gresho and R.L. Sani, On pressure boundary-conditions for the incompressible NavierStokes equations, Int. J. Numer. Methods Fluids 7(10) (1987), 1111-1145.

14. D. Henry, Geometric theory of semilinear parabolic equations, Lecture Notes in Mathematics, vol. 840, Springer-Verlag, Berlin, 1981. MR610244 (83j:35084)

15. J.G Heywood, Auxiliary flux and pressure conditions for Navier-Stokes problems, in: Approximation mathods for Navier-Stokes problems (Proc. Sympos., Univ. Paderborn, Paderborn, 1979), Lecture Notes in Math. vol. 771 (1980), pp. 223-234. MR565999 (83d:35136)

16. J.G. Heywood and R. Rannacher, Finite element approximation of the nonstationary NavierStokes problem. I. Regularity of solutions and second-order error estimates for spatial discretization, SIAM J. Numer. Anal. 19(2) (1982), 275-311. MR650052 (83d:65260)

17. Chang-Yeol Jung and Roger Temam, Numerical approximation of two-dimensional convection-diffusion equations with multiple boundary layers, Int. J. Numer. Anal. Model. 2 (2005), no. 4, 367-408. MR2177629 (2006g:65186)

18. O.A. Ladyženskaja, V.A. Solonnikov, and N.N. Ural'ceva, Linear and quasilinear equations of parabolic type, Translated from the Russian by S. Smith. Translations of Mathematical Monographs, Vol. 23, American Mathematical Society, Providence, RI, 1968. MR0241822 (39:3159b) 
19. O. Ladyženskaya, On the convergence of Fourier series defining a solution of a mixed problem for hyperbolic equations, Doklady Akad. Nauk SSSR (N.S.) 85 (1952), 481-484 (Russian). MR0051412(14:474g)

20. O. A. Ladyženskaya, On solvability of the fundamental boundary problems for equations of parabolic and hyperbolic type, Dokl. Akad. Nauk SSSR (N.S.) 97 (1954), 395-398. MR0073834 $(17: 495 \mathrm{c})$

21. J.-L. Lions, Quelques méthodes de résolution des problèmes aux limites non linéaires, Dunod, 1969. MR0259693 (41:4326)

22. A. Pazy, Semigroups of operators in Banach spaces, Equadiff 82 (Würzburg, 1982), Lecture Notes in Math., vol. 1017, Springer, Berlin, 1983, pp. 508-524. MR726608 (86b:47075)

23. Jeffrey B. Rauch and Frank J. Massey, III, Differentiability of solutions to hyperbolic initialboundary value problems, Trans. Amer. Math. Soc. 189 (1974), 303-318. MR 0340832 (49:5582)

24. Stephen Smale, Smooth solutions of the heat and wave equations, Comment. Math. Helv. 55 (1980), no. 1, 1-12. MR.569242 (83d:35063)

25. R. Temam, Behaviour at time $t=0$ of the solutions of semilinear evolution equations, J. Differential Equations 43 (1982), no. 1, 73-92. MR645638 (83c:35058)

26. _ Navier-Stokes equations, AMS Chelsea Publishing, Providence, RI, 2001, Theory and numerical analysis, Reprint of the 1984 edition.

27. Kevin E. Trenberth, Climate system modeling, Translated from the Russian by S. Smith. Translations of Mathematical Monographs, Vol. 23, Press Syndicate of the University of Cambridge, New York, NY, USA, 1992.

28. V. Thomee and L. Wahlbin, Convergence rates of parabolic difference schemes for non-smooth data, Math.Comp. 28 (1974), 1-13. MR0341889 (49:6635)

Department of Scientific Computing, The Florida State University, Tallahassee, FLORIDA 32306

E-mail address: qchen3@fsu.edu

The Institute for Scientific Computing and Applied Mathematics, Indiana University, Bloomington, Indiana 47405

E-mail address: qinz@indiana.edu

The Institute for Scientific Computing and Applied Mathematics, Indiana University, Bloomington, Indiana 47405

E-mail address: temam@indiana.edu 